Preprint typeset in JHEP style - PAPER VERSION

KUNS-1883

KEK-TH-929

hep-th/0401038

\title{
Nonperturbative studies of fuzzy spheres in a matrix model with the Chern-Simons term
}

\author{
Takehiro Azuma $^{a}$, Subrata Bal ${ }^{a}$, Keiichi Nagao $^{b}$, Jun Nishimura ${ }^{b}$ \\ ${ }^{a}$ Department of Physics, Kyoto University, Kitashirakawa, \\ Kyoto 606-8502, Japan \\ ${ }^{b}$ Institute of Particle and Nuclear Studies, \\ High Energy Accelerator Research Organization (KEK), \\ 1-1 Oho, Tsukuba 305-0801, Japan \\ azuma@gauge.scphys.kyoto-u.ac.jp, subrata@gauge.scphys.kyoto-u.ac.jp, \\ nagao@post.kek.jp, jnishi@post.kek.jp
}

\begin{abstract}
:
Fuzzy spheres appear as classical solutions in a matrix model obtained via dimensional reduction of 3-dimensional Yang-Mills theory with the Chern-Simons term. Well-defined perturbative expansion around these solutions can be formulated even for finite matrix size, and in the case of $k$ coincident fuzzy spheres it gives rise to a regularized $\mathrm{U}(k)$ gauge theory on a noncommutative geometry. Here we study the matrix model nonperturbatively by Monte Carlo simulation. The system undergoes a first order phase transition as we change the coefficient $(\alpha)$ of the Chern-Simons term. In the small $\alpha$ phase, the large $N$ properties of the system are qualitatively the same as in the pure Yang-Mills model $(\alpha=0)$, whereas in the large $\alpha$ phase a single fuzzy sphere emerges dynamically. Various 'multi fuzzy spheres' are observed as meta-stable states, and we argue in particular that the $k$ coincident fuzzy spheres cannot be realized as the true vacuum in this model even in the large $N$ limit. We also perform one-loop calculations of various observables for arbitrary $k$ including $k=1$. Comparison with our Monte Carlo data suggests that higher order corrections are suppressed in the large $N$ limit.
\end{abstract}

Keywords: Matrix Models, Non-Commutative Geometry, Nonperturbative Effects. 


\section{Contents}

1. Introduction 2

2. The Yang-Mills-Chern-Simons matrix model and the fuzzy spheres 4

$\begin{array}{ll}\text { 3. The phase structure } & 7\end{array}$

4. Properties of the single fuzzy sphere $\quad 8$

4.1 The lower critical point and the 'one-loop dominance' 8

$\begin{array}{lll}4.2 & \text { Reproducing an exact result } & 10\end{array}$

5. The eigenvalue distribution of the Casimir operator 11

5.1 The distribution in the fuzzy sphere phase 13

5.2 Space-time picture in the Yang-Mills phase 14

6. Properties of the multi fuzzy spheres $\quad 15$

6.1 Meta-stable states in the thermalization process 15

6.2 The 'life time' of the $k$ coincident fuzzy spheres 16

6.3 The one-loop dominance for the $k$ coincident fuzzy spheres 18

6.4 Comparison of the one-loop effective action 18

7. Summary and discussions $\quad 20$

A. The heat bath algorithm for Monte Carlo simulations 21

B. The one-loop effective action $\quad 22$

$\begin{array}{lll}\text { B.1 The single fuzzy sphere } & 23\end{array}$

B.2 The $k$ coincident fuzzy spheres 24

C. The one-loop calculation of various observables 25

C.1 Propagators and the tadpole 25

$\begin{array}{lll}\text { C.2 One-loop results for various observables } & 26\end{array}$

$\begin{array}{lll}\text { C.3 An alternative derivation } & 27\end{array}$

D. Instability of the $k$ coincident fuzzy spheres $\quad 28$ 


\section{Introduction}

Quantization of gravity is one of the most important problems in particle physics, and lots of attempts have been made so far. String theory, in particular, enables us to quantize small fluctuations of the space-time metric around certain backgrounds, but the nonperturbative determination of the background itself is yet to be done. Such an issue may be addressed by studying matrix models, which are proposed as nonperturbative formulations of string theory. For instance, the IKKT matrix model $[1,2]$, which can be obtained via dimensional reduction of 10-dimensional super Yang-Mills theory, is conjectured to be a nonperturbative definition of type IIB superstring theory in ten dimensions.

In this model the space-time is represented by the eigenvalue distribution of ten bosonic matrices, and if the distribution collapses to a four-dimensional hypersurface, we may naturally understand the dimensionality of our space-time as a result of the nonperturbative dynamics of superstring theory [3]. From the path-integral point of view, this phenomenon may be caused by the phase of the fermion determinant [4], and Monte Carlo results support this mechanism [5]. In Ref. [6] the first evidence for the above scenario has been obtained by calculating the free energy of the space-time with various dimensionality using the Gaussian expansion method up to the 3rd order. Higher-order calculations [7] and the tests of the method itself in simpler models [8] have strengthened the conclusion considerably. Another indication of this phenomenon has been obtained recently from the calculations of the 2loop effective action around fuzzy spheres [9]. See also Refs. [10-14] for related works.

Since the space-time is described by matrices in the IKKT model, it is generically noncommutative. If one expands the model around a D-brane background for instance, one obtains a gauge theory on noncommutative (NC) geometry [15]. 1 This connection was also understood solely in terms of string theory [19], which triggered much interest in the dynamical properties of field theories on $\mathrm{NC}$ geometry in general. It was found that various interesting phenomena which do not have the commutative counterparts occur due to the so-called UV/IR mixing effects [20]. By considering finite-dimensional matrices, one may naturally regularize NC field theories. In particular the twisted reduced models [21], which appeared in history as an equivalent description [22] of large $N$ gauge theories, can be interpreted as a lattice regularization of field theories on a noncommutative torus [23]. This enables nonperturbative studies of various dynamical issues in these theories by Monte Carlo simulation [24-26]. See Ref. [27] for a general review on the dynamics of matrix models in the context of superstring theory and noncommutative geometry.

A different, although closely related, type of regularization is known as the 'fuzzy sphere' [28], which can also be formulated using finite-dimensional matrices. The UV regularization in this case is introduced by putting an upper bound on the angular momentum when one expands a function on the sphere in terms of spherical harmonics. This cutoff procedure is compatible with the 'star-product' of functions which appears in NC geometry. Since the regularized theory does not break the continuous symmetries of the sphere, the

\footnotetext{
${ }^{1}$ For an earlier work on the connection between matrix models and $\mathrm{NC}$ geometry in the context of toroidal compactification, see [16]. A mathematical formulation of field theories on NC geometry is given in Ref. [17]. For a comprehensive review including recent developments, see Ref. [18].
} 
well-known problems in lattice field theory concerning chiral fermions and supersymmetry may be easier to overcome [29]. The first challenge in this direction is to remove the UV/IR mixing effects in the 'continuum limit'.

Being the simplest curved compact noncommutative space, the fuzzy 2-sphere has been studied extensively in the literature. One of the fundamental issues is the construction of the Dirac operator. The one proposed in Refs. [30] does not have the fermion doubling problem $[31,32]$ and it yields the correct chiral anomaly both in the global form [33-35] and in the local form [36]. This Dirac operator, however, breaks chiral symmetry explicitly, and it may therefore be regarded as an analog of the Wilson fermion in lattice gauge theory. On the other hand, an analog of the overlap Dirac operator [37], which satisfies the Ginsparg-Wilson relation [38] and hence preserves modified chiral symmetry [39], is constructed for the free fermion [40] and for general gauge configurations [41, 42]. ${ }^{2}$ In this case the chiral anomaly arises from the measure and the correct results are reproduced in Refs. [41, 48, 49]. Gauge configurations with non-trivial topology have been found for the former Dirac operator $[34,50-52]$ as well as for the latter $[48,53]$. The Seiberg-Witten map [19] has been constructed also on the fuzzy 2-sphere [54].

In string theory fuzzy spheres appear as classical solutions in the presence of an external Ramond-Ramond field [55], and the low-energy effective theory is given by NC Yang-Mills theory with the Chern-Simons term [56] (See also [57]). Classical solutions of the effective theory and their D-brane interpretation have been studied in Ref. [58]. In the matrix-model description of string theory, fuzzy spheres appear as solutions to the classical equation of motion if one adds the Chern-Simons term representing the coupling to the external field [59]. Expanding the bosonic matrices around the classical solution, one obtains a NC gauge theory on fuzzy spheres. The situation is quite analogous to the flat D-brane in matrix models [15] except that the fuzzy sphere can be realized even for finite matrices, which makes various calculations totally well-defined. Studying fuzzy spheres is expected to give us a new insight into the description of curved space-time in matrix models, which is vitally important in the context of quantum gravity. A variety of fuzzy-sphere-like solutions in matrix models have been studied in Refs. [9,59-65]. Fuzzy spheres are also discussed in a matrix model [66] for the M-theory in the so-called pp-wave background [67].

The stability of the fuzzy sphere against quantum fluctuations is a non-trivial important issue, which has been discussed by perturbative calculations $[9,59,64,68]$ and by the Gaussian expansion [65]. Since matrix models typically have various fuzzy-sphere-like solutions, it is important to determine which one describes the true vacuum. Such an issue is related to the dynamical generation of not only the space-time but also the gauge group, since $k$ coincident fuzzy spheres give rise to a $\mathrm{NC}$ gauge theory with the gauge group of rank $k$. The unified treatment of the space-time and the gauge group is one of the advantages of considering noncommutative geometry or the matrix model formulation of string

\footnotetext{
${ }^{2}$ The overlap Dirac operator for general gauge backgrounds has been proposed earlier on a NC torus [43], and used for a construction of chiral gauge theories. The correct chiral anomaly in this case has been reproduced in Ref. [44] for arbitrary even dimensions by using a topological argument [45]. In odd dimensions the analogous Dirac operator is used to define a lattice NC Chern-Simons theory [46] through the parity anomaly [47].
} 
theory.

In this paper we study a matrix model obtained via dimensional reduction of threedimensional Yang-Mills-Chern-Simons theory, which is known to have fuzzy spheres as classical solutions. Unlike previous works we perform fully nonperturbative first-principle calculations using Monte Carlo simulations. We find that the single fuzzy sphere is nonperturbatively stable if the coefficient $(\alpha)$ of the Chern-Simons term is sufficiently large, but it collapses to a 'solid ball' if $\alpha$ is smaller than a critical value. The transition between the two phases is of first order, and we observe a strong hysteresis. Various 'multi fuzzy spheres', which are also classical solutions of the model, are observed as meta-stable states, and we argue in particular that the $k$ coincident fuzzy spheres cannot be realized as the true vacuum even in the large $N$ limit. We also perform one-loop calculations of various observables for arbitrary $k$ including $k=1$. Comparison with our Monte Carlo data suggests that higher order corrections are suppressed in the large $N$ limit.

The rest of the paper is organized as follows. In Section 2 we define the model and review how fuzzy spheres appear in this model as classical solutions. In Section 3 we investigate the phase diagram of the model and demonstrate the existence of the first order phase transition. In Section 4 we discuss the properties of the single fuzzy sphere. In Section 5 we study the geometrical structure of the dominant configurations in each phase. In Section 6 we show how various multi fuzzy spheres appear as meta-stable states and study their properties. Section 7 is devoted to a summary and discussions. In Appendix A we comment on the algorithm used for our Monte Carlo simulations. In Appendices B and $\mathrm{C}$ we give a self-contained derivation of the one-loop results for the effective potential as well as various observables. In Appendix D we discuss the instability of the $k$ coincident fuzzy spheres.

\section{The Yang-Mills-Chern-Simons matrix model and the fuzzy spheres}

The model we study in this paper is defined by the partition function [59]

$$
\begin{aligned}
& Z=\int \mathrm{d} A \mathrm{e}^{-S} \\
& S=N \operatorname{tr}\left(-\frac{1}{4}\left[A_{\mu}, A_{\nu}\right]^{2}+\frac{2}{3} i \alpha \epsilon_{\mu \nu \rho} A_{\mu} A_{\nu} A_{\rho}\right),
\end{aligned}
$$

where $A_{\mu}(\mu=1,2,3)$ are $N \times N$ traceless Hermitian matrices. The integration measure $\mathrm{d} A$ is defined by $\mathrm{d} A=\prod_{a=1}^{N^{2}-1} \prod_{\mu=1}^{3} \frac{d A_{\mu}^{a}}{\sqrt{2 \pi}}$, where $A_{\mu}^{a}$ are the coefficients in the expansion $A_{\mu}=\sum_{a} A_{\mu}^{a} t^{a}$ with respect to the $\mathrm{SU}(N)$ generators $t^{a}$ normalized as $\operatorname{tr}\left(t^{a} t^{b}\right)=\frac{1}{2} \delta^{a b}$. The coefficient of the first term in (2.2) is fixed, but this does not spoil any generality since one can always rescale $A_{\mu}$ to bring the action into the present form.

This model may be regarded as the zero-volume limit of $\mathrm{SU}(N)$ Yang-Mills theory with the Chern-Simons term in the three-dimensional Euclidean space, and it has the $\mathrm{SO}(3)$ rotational symmetry as well as the $\mathrm{SU}(N)$ symmetry $A_{\mu} \rightarrow U A_{\mu} U^{\dagger}$, where $U \in \mathrm{SU}(N)$. There are some points to note here, though. The parameter $\alpha$ in (2.2) is chosen to be real in order for fuzzy spheres to be classical solutions of the model. As a result the Chern-Simons 
term in the action (2.2) is real unlike the counterpart in ordinary field theory, where it is purely imaginary and therefore poses a severe technical problem in Monte Carlo simulation. Note also that $\alpha$ may take arbitrary real number without breaking any symmetries unlike the ordinary Chern-Simons theory, where the coefficient is quantized for the invariance under topologically nontrivial gauge transformations. Since the model has the duality $\alpha \mapsto-\alpha$ associated with the parity transformation $A_{\mu} \mapsto-A_{\mu}$, we restrict ourselves to $\alpha>0$ throughout this paper without loss of generality.

The pure Yang-Mills model $(\alpha=0)$ and its obvious generalization to $D$ dimensions with $D$ matrices $A_{\mu}(\mu=1, \cdots, D)$ have been studied by many authors. In particular the large $N$ dynamics of the model have been studied by the $1 / D$ expansion and Monte Carlo simulations [69]. The partition function was conjectured [71] and proved [72] to be finite for $N>D /(D-2)$. (See Refs. [73,74] for the supersymmetric case.) The partition function in the presence of the Chern-Simons term has been studied analytically for $N=2$ [75], and it turned out to be convergent in the supersymmetric case, but not in the bosonic case. It is also proved that adding a Myers term (the Chern-Simons term in the present case) does not affect the convergence as far as the original path integral converges absolutely [76], which means in particular that the partition function (2.1) is convergent for $N \geq 4$. Note that this statement holds despite the fact that the classical action (2.2) is unbounded from below for $\alpha \neq 0$.

The classical equation of motion in the present model reads

$$
\left[A_{\nu},\left[A_{\nu}, A_{\mu}\right]\right]+i \alpha \epsilon_{\mu \nu \rho}\left[A_{\nu}, A_{\rho}\right]=0 .
$$

The simplest type of solutions is given by the commutative matrices, which can be brought into the diagonal form

$$
A_{\mu}=\operatorname{diag}\left(x_{\mu}^{(1)}, x_{\mu}^{(2)}, \cdots, x_{\mu}^{(N)}\right)
$$

by an appropriate $\mathrm{SU}(N)$ transformation. This type of solutions exists also at $\alpha=0$, and the one-loop effective action around it has been calculated in Ref. [69]. For $\alpha \neq 0$ the one-loop effective action reads [59]

$$
\Gamma_{1-\text { loop }}=\sum_{i<j}\left[\log \left\{\left(x_{\mu}^{(i)}-x_{\mu}^{(j)}\right)^{2}\right\}+\log \left\{1-\frac{4 \alpha^{2}}{\left(x_{\mu}^{(i)}-x_{\mu}^{(j)}\right)^{2}}\right\}\right],
$$

where the second term represents the additional piece for $\alpha \neq 0$. The resulting attractive force between $x_{\mu}^{(i)}$ makes their distribution shrink until the perturbative calculation becomes no more valid [69].

When $\alpha$ is nonzero, there exists another type of solutions given by

$$
A_{\mu}=\alpha L_{\mu}
$$

where $L_{\mu}(\mu=1,2,3)$ satisfy

$$
\left[L_{\mu}, L_{\nu}\right]=i \epsilon_{\mu \nu \rho} L_{\rho} .
$$


Since the equation (2.7) is nothing but the $\mathrm{SU}(2)$ Lie algebra, each of its $N$-dimensional representation yields a classical solution. We denote the $n$-dimensional irreducible representation of the algebra by $L_{\mu}^{(n)}$, which can be characterized by the identity

$$
\sum_{\mu}\left(L_{\mu}^{(n)}\right)^{2}=\frac{1}{4}\left(n^{2}-1\right) \mathbf{1}_{n}
$$

Let us consider the case in which $L_{\mu}$ itself is given by the irreducible representation. The corresponding classical solution

$$
A_{\mu}=\alpha L_{\mu}^{(N)}
$$

satisfies

$$
\begin{aligned}
\sum_{\mu}\left(A_{\mu}\right)^{2} & =R^{2} \mathbf{1}_{N}, \\
R & =\frac{1}{2} \alpha \sqrt{N^{2}-1} .
\end{aligned}
$$

If we neglect for the moment the fact that $A_{\mu}$ are noncommutative, eq. (2.10) implies that all the $N$ eigenvalues of $A_{\mu}$ are distributed on a sphere centered at the origin with the radius $R$. Since $A_{\mu}$ are noncommutative in reality, this classical solution is called the fuzzy 2 -sphere (In this paper we refer to it as the 'single fuzzy sphere').

If we consider a general representation, the corresponding classical solution can be brought into the form

$$
A_{\mu}=\alpha\left(\begin{array}{llll}
L_{\mu}^{\left(n_{1}\right)} & & & \\
& L_{\mu}^{\left(n_{2}\right)} & & \\
& & \ddots & \\
& & & L_{\mu}^{\left(n_{k}\right)}
\end{array}\right)
$$

by an appropriate $\mathrm{SU}(N)$ transformation, where

$$
\sum_{a=1}^{k} n_{a}=N
$$

This solution satisfies

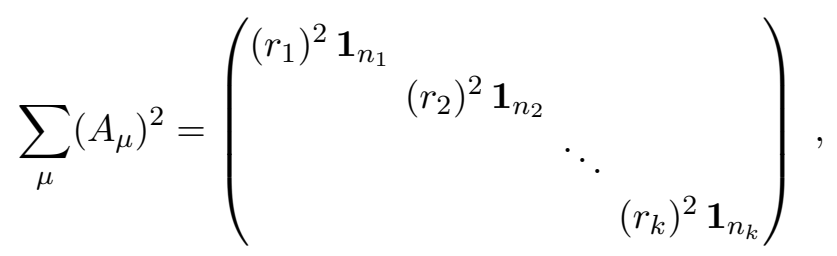

where

$$
r_{a}=\frac{1}{2} \alpha \sqrt{\left(n_{a}\right)^{2}-1}
$$

meaning that $n_{a}$ eigenvalues of $A_{\mu}$ are distributed on a sphere centered at the origin with the radius $r_{a}$. In the $k \geq 2$ case we call the solution (2.12) 'multi fuzzy spheres'. 
The particular case of 'multi fuzzy spheres' that we will focus on later corresponds to taking $n_{1}=\cdots=n_{k} \equiv n$, and therefore $N=n \cdot k$ due to (2.13). The radius (2.15) for each sphere becomes equal, and it is proportional to $1 / k$ at large $N$. Expansion of the model around such a configuration, which describes $k$ coincident fuzzy spheres, gives rise to a NC gauge theory on the fuzzy sphere with the gauge group of rank $k$ [59]. This statement holds also for $k=1$, which corresponds to the single fuzzy sphere.

We study the matrix model (2.2) by Monte Carlo simulations using the heat-bath algorithm, which was adopted also in the study of the pure Yang-Mills model $(\alpha=0)$ in Ref. [69]. See Appendix A for more details.

\section{The phase structure}

In this Section we study the phase structure of the model by calculating fundamental quantities such as $\langle S\rangle$ and $\left\langle\frac{1}{N} \operatorname{tr}\left(A_{\mu}\right)^{2}\right\rangle$ by Monte Carlo simulations. Here we take the initial configuration to be either of the following two.

$$
A_{\mu}^{(0)}= \begin{cases}\alpha L_{\mu}^{(N)} & (\text { the single fuzzy sphere start }) \\ 0 & (\text { the zero start })\end{cases}
$$

Fig. 1 shows the results plotted against $\alpha$ for $N=8,16,24$. The one-loop results obtained from the perturbative expansion around the single fuzzy sphere are plotted for comparison.
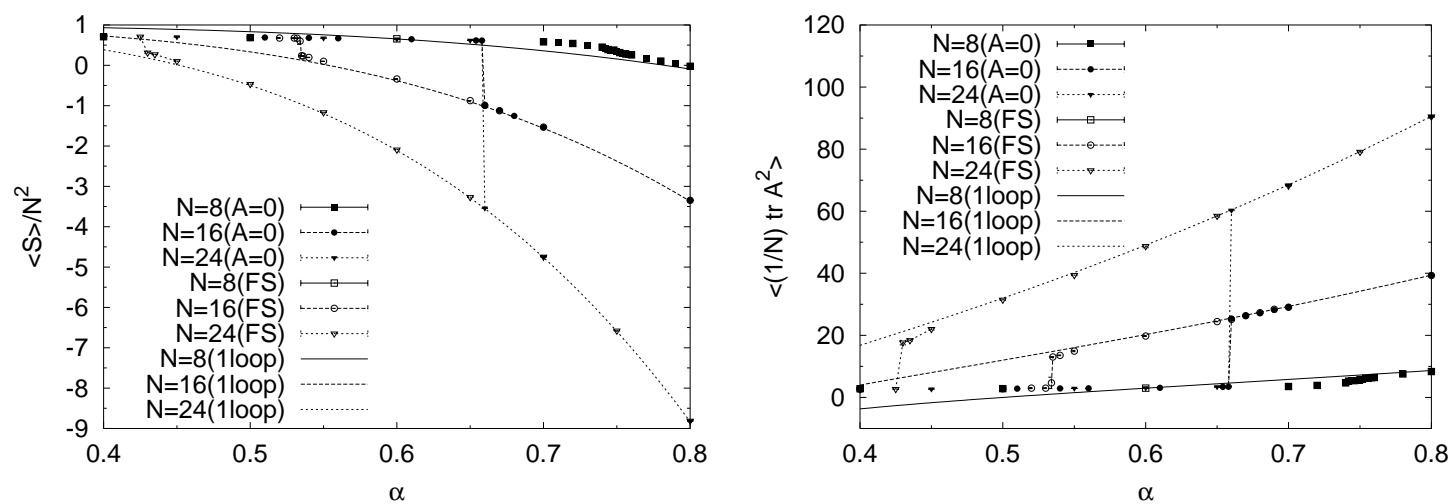

Figure 1: The observables $\frac{1}{N^{2}}\langle S\rangle$ (left) and $\left\langle\frac{1}{N} \operatorname{tr} A_{\mu}^{2}\right\rangle$ (right) are plotted against $\alpha$ for $N=8,16,24$. The open symbols represent the single fuzzy sphere start, whereas the closed symbols represent the zero start. We also plot the one-loop results for the single fuzzy sphere at each $N$ for comparison.

The Monte Carlo results depend on the initial configuration in the intermediate region of $\alpha$ for $N \geq 16$, and we observe discontinuities at

$$
\alpha= \begin{cases}\alpha_{\mathrm{cr}}^{(\mathrm{l})} \sim \frac{2.1}{\sqrt{N}} & \text { (for the single fuzzy sphere start) } \\ \alpha_{\mathrm{cr}}^{(\mathrm{u})} \sim 0.66 & \text { (for the zero start) },\end{cases}
$$

which we call the lower/upper critical points, respectively. This clearly demonstrates the existence of a first order phase transition. 
Note that the large $N$ behavior of the lower critical point is different from the upper critical point. If we naively apply the formula (3.2) to $N=8$, we obtain $\alpha_{\mathrm{cr}}^{(1)}=\frac{2.1}{\sqrt{8}}=0.742$, which is actually larger than $\alpha_{\mathrm{cr}}^{(\mathrm{u})}$. What happens in reality is that the hysteresis simply disappears for $N=8$ as seen in Fig. 1 .

In the small $\alpha$ phase the results do not depend much on $\alpha$. In particular the large $N$ behavior of each observable is qualitatively the same as in the pure Yang-Mills model [69] $(\alpha=0)$, i.e., $\frac{1}{N^{2}}\langle S\rangle \sim \mathrm{O}(1)$ and $\left\langle\frac{1}{N} \operatorname{tr}\left(A_{\mu}\right)^{2}\right\rangle \sim \mathrm{O}(1)$. We will call this phase the 'YangMills phase'. In the large $\alpha$ phase the results agree very well with the one-loop perturbative calculations around the single fuzzy sphere, which we elucidate further in Section 4 . We will call this phase the 'fuzzy-sphere phase'. When we perform simulations with the zero start for $\alpha>\alpha_{\mathrm{cr}}^{(\mathrm{u})}$, we observe various multi fuzzy spheres as meta-stable states in the thermalization process (See Section 6.1).

\section{Properties of the single fuzzy sphere}

In this Section we study various properties of the single fuzzy sphere by Monte Carlo simulations using (2.9) as the initial configuration.

\subsection{The lower critical point and the 'one-loop dominance'}

Here we calculate various observables by Monte Carlo simulations, and compare the results with the one-loop perturbative calculations. We determine the lower critical point, and show that our Monte Carlo data above the critical point agree very well with the one-loop results.

Let us first summarize the results of one-loop calculations. For brevity we introduce the notation

$$
\begin{aligned}
F_{\mu \nu} & =i\left[A_{\mu}, A_{\nu}\right], \\
M & =\frac{2}{3 N} i \epsilon_{\mu \nu \lambda} \operatorname{tr}\left(A_{\mu} A_{\nu} A_{\lambda}\right),
\end{aligned}
$$

so that the action reads $S=N^{2}\left(\frac{1}{4 N} \operatorname{tr} F^{2}+\alpha M\right)$. The leading large $N$ behavior of various observables is given by

$$
\begin{aligned}
\frac{1}{N^{2}}\langle S\rangle_{1-\text { loop }} & \simeq-\frac{\tilde{\alpha}^{4}}{24}+1 \\
\frac{1}{N}\left\langle\frac{1}{N} \operatorname{tr}\left(A_{\mu}\right)^{2}\right\rangle_{1-\text { loop }} & \simeq \frac{\tilde{\alpha}^{2}}{4}-\frac{1}{\tilde{\alpha}^{2}} \\
\left\langle\frac{1}{N} \operatorname{tr}\left(F_{\mu \nu}\right)^{2}\right\rangle_{1-\text { loop }} & \simeq \frac{\tilde{\alpha}^{4}}{2}+0, \\
\frac{1}{\sqrt{N}}\langle M\rangle_{1-\text { loop }} & \simeq-\frac{\tilde{\alpha}^{3}}{6}+\frac{1}{\tilde{\alpha}},
\end{aligned}
$$

where we have introduced the rescaled parameter

$$
\tilde{\alpha}=\alpha \sqrt{N} .
$$



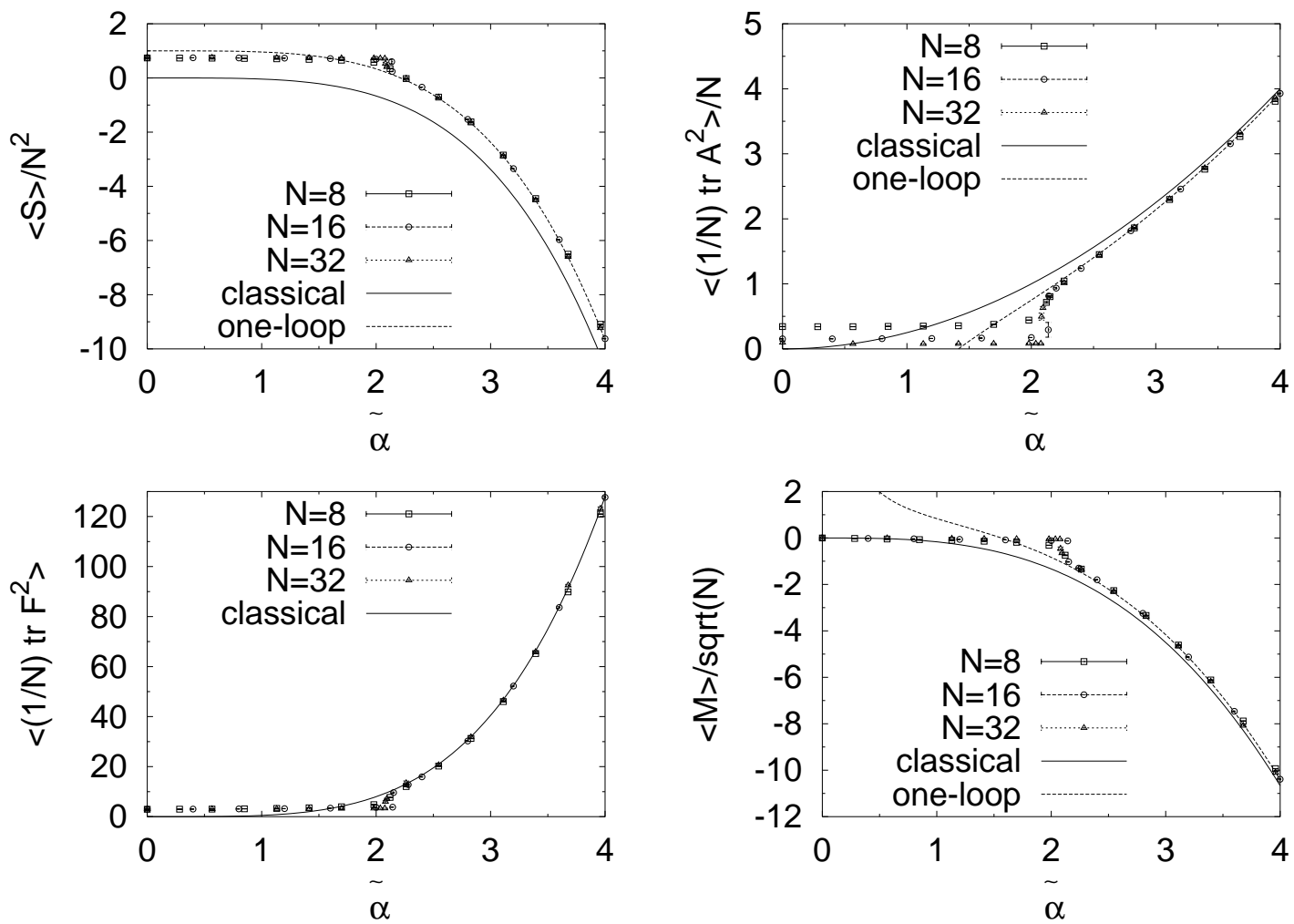

Figure 2: Various observables are plotted against $\tilde{\alpha}=\alpha \sqrt{N}$ for $N=8,16,32$ with the single fuzzy sphere start. The solid lines represent the classical results, whereas the dashed lines represent the results including the one-loop corrections.

In $(4.3) \sim(4.6)$ the first term represents the classical result, while the second term represents the one-loop correction (See Appendix C for derivation).

In Fig. 2 we plot our Monte Carlo results for these quantities against $\tilde{\alpha}$. All the observables exhibit a discontinuity at

$$
\tilde{\alpha}=\tilde{\alpha}_{\mathrm{cr}}^{(\mathrm{l})} \simeq 2.1
$$

In terms of the original parameter $\alpha$, it corresponds to the lower critical point given in (3.2).

The results above the lower critical point scale with $N$, and moreover they agree very well with the perturbative results including the one-loop corrections. For $\left\langle\frac{1}{N} \operatorname{tr}\left(F_{\mu \nu}\right)^{2}\right\rangle$, the data agree even with the classical result due to the accidental cancellation of the one-

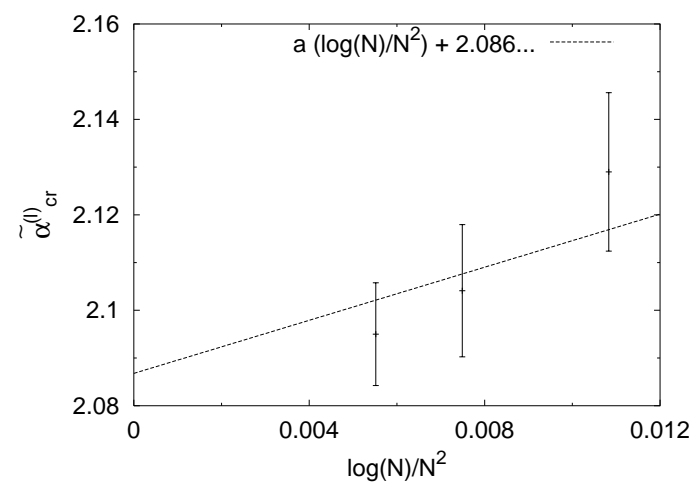

Figure 3: The lower critical point $\tilde{\alpha}_{\mathrm{cr}}^{(1)}$ is plotted for $N=16,20,24$. The dashed line represents a fit to $\tilde{\alpha}_{\mathrm{cr}}^{(1)}=\left(\frac{8}{3}\right)^{3 / 4}+a \frac{\log N}{N^{2}}$, where $a=2.781$. 
loop correction (4.5). These results suggest that the higher order corrections are suppressed at large $N$ for fixed $\tilde{\alpha}$.

In fact it turns out that the lower critical point (4.8) can be reproduced from the effective potential for the scalar mode on the fuzzy sphere [70] as ${ }^{3}$

$$
\tilde{\alpha}_{\mathrm{cr}}^{(1)}=\left(\frac{8}{3}\right)^{3 / 4}+a \frac{\log N}{N^{2}} .
$$

In Fig. 3 we plot the lower critical point $\tilde{\alpha}_{\text {cr }}^{(1)}$ determined by simulations for $N=8,16,24$, where the 'error bars' represent the range of $\tilde{\alpha}$ for which we observe two-state signals. Our results are consistent with the large $N$ asymptotic behavior (4.9).

\subsection{Reproducing an exact result}

In this Section we consider an exact result, which can be used as a check of Monte Carlo simulations and the 'one-loop dominance' discussed in the previous Section. Let us note that the expectation value of the observable

$$
K=\frac{1}{N} \operatorname{tr}\left(F_{\mu \nu}\right)^{2}+3 \alpha M
$$

is given exactly by

$$
\langle K\rangle=3\left(1-\frac{1}{N^{2}}\right) \equiv K_{0}
$$

for arbitrary $\alpha$ and $N$. This identity has been studied also in the $\alpha=0$ case [69].

The exact result (4.11) can be derived from the invariance of the partition function (2.1) under the change of integration variables $A_{\mu} \mapsto(1+\epsilon) A_{\mu}$. Alternatively one may derive it from

$$
0=\int \mathrm{d} A \frac{\partial}{\partial A_{\mu}^{a}}\left\{\operatorname{tr}\left(t^{a} A_{\mu}\right) \mathrm{e}^{-S}\right\},
$$

which is one of the Schwinger-Dyson equations in this model.

In Fig. 4 we show our results for the ratio $\frac{\langle K\rangle}{K_{0}}$. All the data for various $\tilde{\alpha}$ and $N$ are consistent with ' 1 ' within error bars, which demonstrates the validity of our simulation.

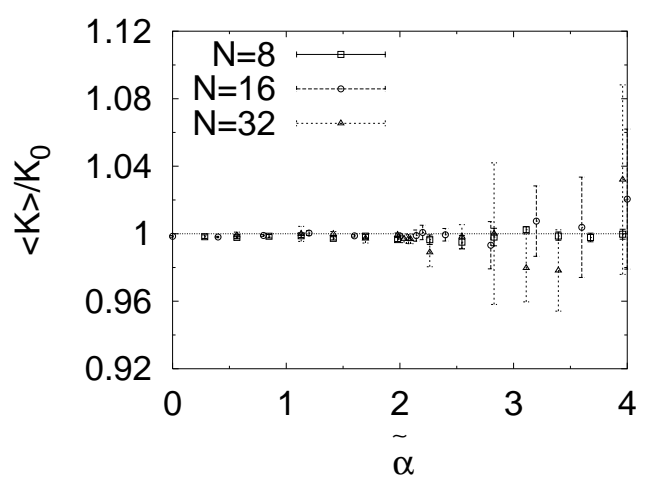

Figure 4: The ratio $\frac{\langle K\rangle}{K_{0}}$ is plotted against $\tilde{\alpha}$ for $N=8,16,32$ with the single fuzzy sphere start. The horizontal line corresponds to the exact result.

The exact result (4.11) is consistent with our assertion that higher loop corrections are suppressed at large $N$ in the fuzzy sphere phase. Using (4.5) and (4.6), we obtain

$$
\begin{aligned}
\langle K\rangle_{1-\text { loop }} & =\left\langle\frac{1}{N} \operatorname{tr} F^{2}\right\rangle_{1-\text { loop }}+3 \alpha\langle M\rangle_{1-\text { loop }} \\
& =\frac{\tilde{\alpha}^{4}}{2}+3 \tilde{\alpha}\left(-\frac{\tilde{\alpha}^{3}}{6}+\frac{1}{\tilde{\alpha}}\right)=3,
\end{aligned}
$$

\footnotetext{
${ }^{3}$ This was informed to us by Denjoe O'Connor after J.N. gave a seminar on the main results of this paper including (4.8) at Dublin Institute for Advanced Studies.
} 
which agrees with the exact result (4.11) in the large $N$ limit. We also notice that as $\tilde{\alpha}$ becomes large, there is a huge cancellation between the first term $\left\langle\frac{1}{N} \operatorname{tr}\left(F_{\mu \nu}\right)^{2}\right\rangle$ and the second term $3 \alpha\langle M\rangle$. Namely the large $\tilde{\alpha}$ behavior of each term is $\mathrm{O}\left(\tilde{\alpha}^{4}\right)$, but the leading term cancels exactly leaving behind an $\mathrm{O}(1)$ quantity. This is reflected in Fig. 4, where the error bars become much larger at $\tilde{\alpha}>\tilde{\alpha}_{\mathrm{cr}}^{(\mathrm{l})}$. Such a cancellation is absent in the Yang-Mills phase since the second term $3 \alpha\langle M\rangle$ is close to zero as one can see from Fig. 2.

\section{The eigenvalue distribution of the Casimir operator}

In the previous Section we have seen that our Monte Carlo results in the fuzzy sphere phase agree very well with the one-loop calculations around the single fuzzy sphere. Here we show more directly that the dominant configurations in this phase indeed have the geometry of the 2 -sphere.

For that purpose it is convenient to consider the distribution

$$
f(x)=\frac{1}{N} \sum_{j=1}^{N}\left\langle\delta\left(x-\lambda_{j}\right)\right\rangle,
$$

where $\lambda_{j}(j=1, \cdots, N)$ are $N$ real positive eigenvalues of the Casimir operator

$$
Q=\sum_{\mu}\left(A_{\mu}\right)^{2}
$$

Note that the set of eigenvalues $\left\{\lambda_{i}\right\}$ is invariant under both $\mathrm{SO}(3)$ and $\mathrm{SU}(N)$ transformations. Within the classical approximation, the distribution $f(x)$ is given by

$$
f(x)=\delta\left(x-R^{2}\right)
$$

for the single fuzzy sphere (2.9), where $R$ is defined in (2.11). Similarly for the multi fuzzy spheres (2.12) we get

$$
f(x)=\sum_{a=1}^{k}\left(\frac{n_{a}}{N}\right) \delta\left(x-r_{a}^{2}\right),
$$

where $r_{a}$ is defined in (2.15). This will play an important role in identifying various multi fuzzy spheres in Section 6.

Fig. 5 shows the results for $\alpha=0.5<\alpha_{\mathrm{cr}}^{(\mathrm{l})}$ (left) and $\alpha=1.0,2.0>\alpha_{\mathrm{cr}}^{(\mathrm{u})}$ (right) at $N=16$. The vertical lines represent the delta function corresponding to the classical result (5.3) for the single fuzzy sphere. At $\alpha=1.0$ and 2.0 the measured distribution is close to the classical result. Slight deviations such as the smearing and the shift can be understood as quantum effects, as we will see in Section 5.1. Thus the dominant configurations are not given exactly by the single fuzzy sphere (2.9), but they still preserve the geometrical structure of a 2 -sphere with a slightly smaller radius and with a finite width.

The result for $\alpha=0.5$, on the other hand, deviates drastically from the classical result (5.3). In Section 5.2 we will discuss how the observed distribution can be understood qualitatively by considering the diagonal configuration (2.4) and the effective action (2.5). 

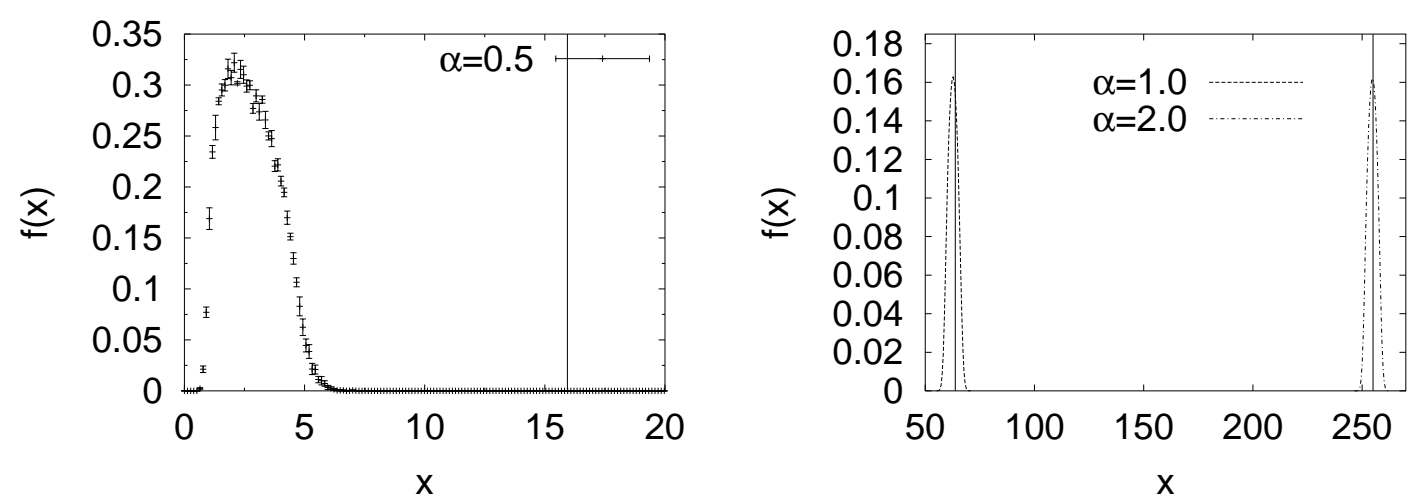

Figure 5: The eigenvalue distribution $f(x)$ of the Casimir operator (5.2) is plotted for $N=16$ at $\alpha=0.5<\alpha_{\mathrm{cr}}^{(\mathrm{l})}$ (left) and $\alpha=1.0,2.0>\alpha_{\mathrm{cr}}^{(\mathrm{u})}$ (right).

At the intermediate values of $\alpha$ we observe a two-state signal, and as a result the measured distribution is given by the superposition of the two types of distribution described above. This is expected from the first-order nature of the phase transition revealed in Section 3.
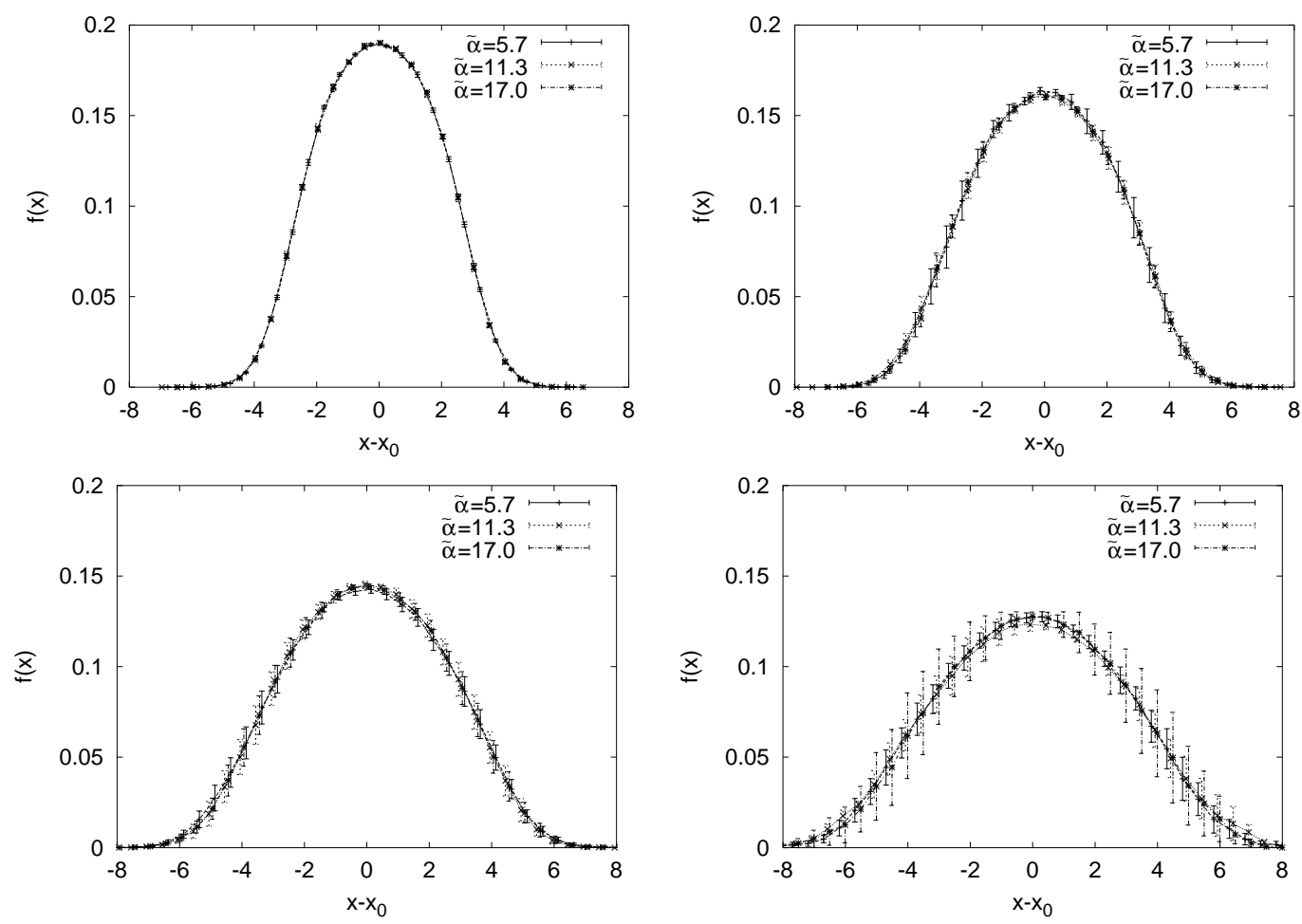

Figure 6: The eigenvalue distribution $f(x)$ of the Casimir operator (5.2) is plotted against $x-x_{0}$ for $N=8$ (upper left), $N=16$ (upper right), $N=32$ (lower left) and $N=64$ (lower right), respectively. At each $N$ the results for different $\tilde{\alpha}$ lie on top of each other. 


\subsection{The distribution in the fuzzy sphere phase}

Here we discuss the properties of the distribution $f(x)$ in the fuzzy sphere phase in more detail, and show that they can be understood by the one-loop dominance.

Let us start with the position of the peak, which may be defined by

$$
x_{0} \equiv \int_{0}^{\infty} \mathrm{d} x x f(x)=\left\langle\frac{1}{N} \operatorname{tr}\left(A_{\mu}\right)^{2}\right\rangle \text {. }
$$

This quantity is actually studied in Section 4.1 and it agrees with the one-loop result (4.4) for the fuzzy sphere. Note that the one-loop correction is negative, which implies that the peak moves toward the origin compared with the classical result (5.3). Also the one-loop correction is proportional to $\frac{1}{\tilde{\alpha}^{2}}$, meaning that the shift of the peak becomes more pronounced at

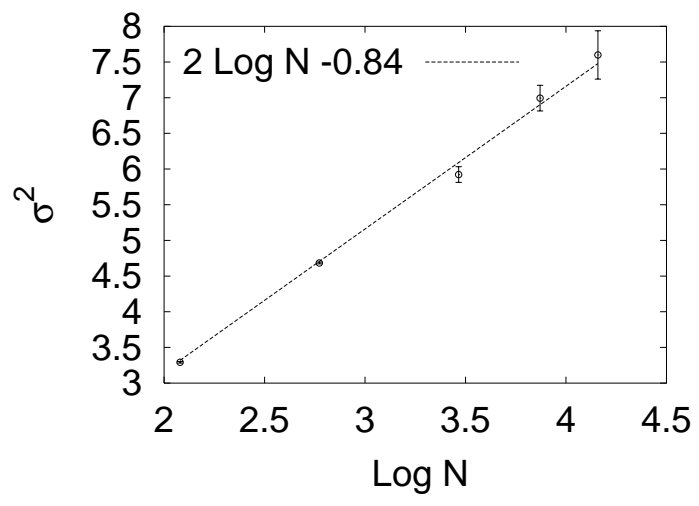

Figure 7: The variance $\sigma^{2}$ of the distribution $f(x)$ is plotted for $N=8,16,32,48,64$. The straight line is a fit to $\sigma^{2} \sim 2 \log N+b$, where $b=-0.84$. smaller $\tilde{\alpha}$. These properties are indeed observed in Fig. 5 (right). An interesting point to note here is that the one-loop result (4.4) for $\frac{1}{N}\left\langle\frac{1}{N} \operatorname{tr}\left(A_{\mu}^{2}\right)\right\rangle$ becomes zero at $\tilde{\alpha}=\sqrt{2}$. Assuming the one-loop dominance, we may suspect that nonperturbative effects should become non-negligible as we decrease $\tilde{\alpha}$, which invalidates the perturbation theory before $\tilde{\alpha}$ reaches $\sqrt{2}$. This is indeed what happens in reality (Note that $\tilde{\alpha}_{\text {cr }}^{(1)}=2.1>\sqrt{2}$ ).

Having understood the position of the peak, let us turn to the shape of the distribution. In Fig. 6 we plot the distribution $f(x)$ against $x-x_{0}$ for various $\tilde{\alpha}$ at $N=8,16,32,64$. The results for different $\tilde{\alpha}$ collapse to a single curve, which means that the shape of the distribution does not depend on $\tilde{\alpha}$. Furthermore the distribution turns out to be symmetric around $x=x_{0}$. These properties can be understood from the 'one-loop dominance'.

Among the quantities that characterize the shape of distributions, let us focus on the width $\sigma$, which may be defined by

$$
\begin{aligned}
\sigma^{2} & \equiv \int_{0}^{\infty} \mathrm{d} x\left(x-x_{0}\right)^{2} f(x) \\
& =\left\langle\frac{1}{N} \operatorname{tr}\left(A_{\mu}^{2}\right)^{2}\right\rangle-\left\langle\frac{1}{N} \operatorname{tr}\left(A_{\mu}\right)^{2}\right\rangle^{2} .
\end{aligned}
$$

We can calculate it by the perturbative expansion around the single fuzzy sphere. Since the classical result is zero, it starts from the one-loop contribution, which is given by

$$
\sigma^{2}=2 \log N+\mathrm{O}(1)
$$

at large $N$ (See Appendix $\mathrm{C}$ for a derivation). The perturbative result is independent of $\alpha$ at this order, but higher order corrections may yield terms proportional to $\left(\frac{1}{\alpha^{4}}\right)^{n}$ $(n=1,2, \cdots)$. The $\tilde{\alpha}$ independence observed in our Monte Carlo data can therefore be 
understood by the one-loop dominance at large $N$. In Fig. 7 we plot the variance (5.6) obtained from simulations with $N=8,16,32,48,64$. Taking account of the $\mathrm{O}(1)$ term, we find that the large $N$ behavior agrees very well with the one-loop result (5.7).

\subsection{Space-time picture in the Yang-Mills phase}

Let us move on to the result for the eigenvalue distribution $f(x)$ in the Yang-Mills phase, which is shown in Fig. 5 (left). If the dominant configurations in this phase were given by the diagonal configurations (2.4), the eigenvalues of the Casimir operator would be given by $\sum_{\mu}\left(x_{\mu}^{(j)}\right)^{2}(j=1, \cdots, N)$. Therefore the eigenvalue distribution $f(x)$ of the Casimir operator (5.2) would be related to the radial density distribution $\rho(r)$ of the $N$ space-time points $x_{\mu}^{(j)}(j=1, \cdots, N)$ by

$$
f(x) \mathrm{d} x=\rho(r) 4 \pi r^{2} \mathrm{~d} r
$$

where $x=r^{2}$. From (5.8) we get

$$
\rho(r)=\frac{1}{2 \pi r} f\left(r^{2}\right)
$$

In reality the dominant configurations are not diagonal up to the $\mathrm{SU}(N)$ symmetry, but we may still define the 'radial density distribution' $\rho(r)$ through (5.9). In Fig. 8 we plot $\rho(r)$ for $N=8,16,32$ at $\alpha=0.0$ and for $N=32$ at $\alpha=0.0, \cdots, 0.6$. For $\alpha \geq 0.4$ we have to use the zero start in order to stay in the Yang-Mills phase. The distribution at $\alpha=0$ converges at large $N$, and this is confirmed also for $\alpha \neq 0$.
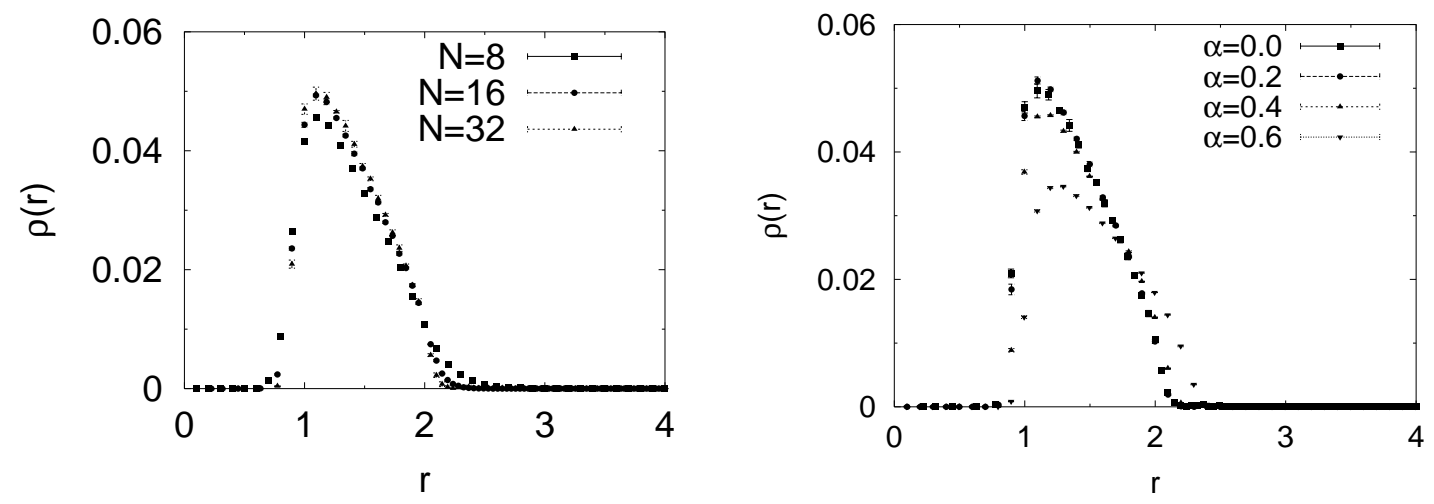

Figure 8: The 'radial density distribution' $\rho(r)$ is plotted for $N=8,16,32$ at $\alpha=0.0$ (left) and for $N=32$ at $\alpha=0.0,0.2,0.4,0.6$ (right).

An interesting point to note here is that the distribution $\rho(r)$ has an empty region $0 \leq r \leq r_{0}$, where $r_{0}=0.8 \sim 0.9$. This may be understood as follows. If there exists an eigenvector of the Casimir operator $Q=\sum_{\mu}\left(A_{\mu}\right)^{2}$ whose eigenvalue is close to zero, the same vector is an approximate eigenvector of $A_{1}, A_{2}$ and $A_{3}$ separately with eigenvalues which are also close to zero. Existence of such a vector is not allowed, however, since a generic configuration $\left\{A_{\mu} ; \mu=1,2,3\right\}$ that appears in the ensemble does not commute with 
each other. The appearance of the empty region can thus be understood by the 'uncertainty principle'. Note that the scale of noncommutativity is of $\mathrm{O}(1)$ since $\left\langle\frac{1}{N} \operatorname{tr} F_{\mu \nu}^{2}\right\rangle \sim \mathrm{O}(1)$ in the Yang-Mills phase. Therefore the size $r_{0}$ of the empty region should be of $\mathrm{O}(1)$ as well, which is in agreement with our Monte Carlo results.

Except for this empty region, the distribution $\rho(r)$ decreases monotonously. This behavior can be understood by the one-loop effective action (2.5), which gives rise to an attractive potential between any pair of space-time points. The rapid fall of $\rho(r)$ at large $r$ is consistent with the results obtained for $\alpha=0$ [77]. Note, however, that the precise form of the distribution cannot be understood solely from the perturbation theory around the diagonal configuration (2.4), since the higher order corrections become non-negligible as the space-time points come closer to each other due to the one-loop potential (2.5). For instance the distribution $\rho(r)$ is seen to expand to larger $r$ as $\alpha$ increases, but this behavior does not agree with the naive expectation from the one-loop effective action (2.5), where the second term adds to the attractive potential. We may understand it from a nonperturbative point of view, however, since the Chern-Simons term, which can be more negative for larger $A_{\mu}$, effectively enhances configurations with a larger extent.

\section{Properties of the multi fuzzy spheres}

As we described in Section 2 the model has various multi fuzzy spheres as classical solutions in addition to the single fuzzy sphere. The aim of this Section is to see how they appear in simulations and to study their properties.

\subsection{Meta-stable states in the thermalization process}
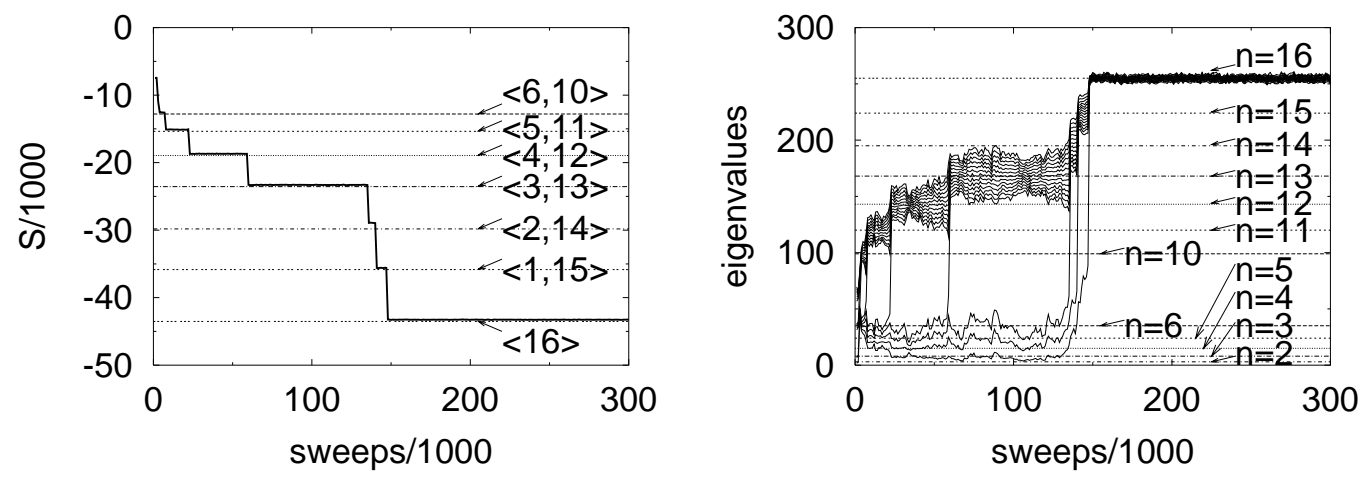

Figure 9: The history of the action $S$ (left) and the eigenvalues of the Casimir operator (right) are plotted for $N=16, \alpha=2.0$ with the zero start. The horizontal lines represent the classical results for the action and the radius squared, respectively.

Let us note first that the action for the classical solution (2.12) is given by

$$
S=-\frac{\alpha^{4} N}{24} \sum_{a=1}^{k}\left(n_{a}^{3}-n_{a}\right)
$$


which becomes minimum for the single fuzzy sphere $(k=1)$ (Notice the constraint $(2.13)$ ). Therefore at the classical level the multi fuzzy spheres are expected to appear only as meta-stable states. ${ }^{4}$

Indeed we do find them in the process of thermalization. We have performed simulations at $N=16$ and $\alpha=2.0>\alpha_{\mathrm{cr}}^{(\mathrm{u})}$ with the zero start. Fig. 9 shows the history of the action $S$ and the eigenvalues of the Casimir operator (5.2). We have introduced a short-hand notation $\left\langle n_{1}, n_{2}\right.$, $\left.\cdots, n_{k}\right\rangle$ for the classical solution (2.12). One sweep is defined as updates of all the elements of the matrices $A_{\mu}$ by the heat bath algorithm (See Appendix A). We see many plateaus before we reach the single fuzzy sphere after 150,000 sweeps. These meta-stable states can be identified with the multi fuzzy spheres by comparing the

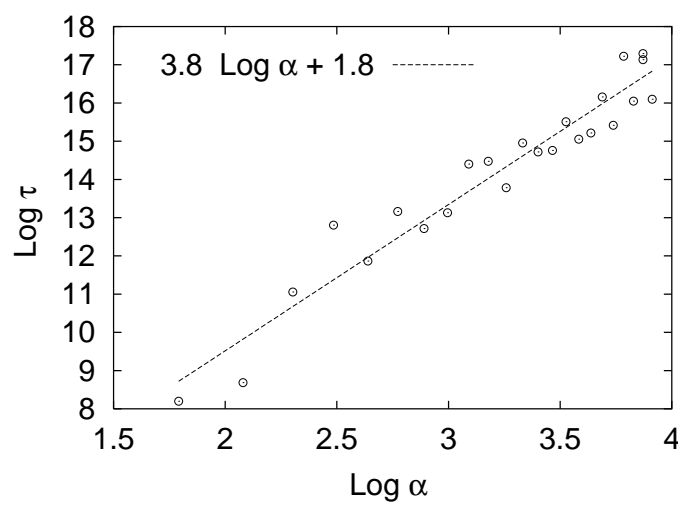

Figure 10: The 'life time' $(\tau)$ of the multi fuzzy spheres $\langle 3,5\rangle$ for $N=8$ is shown as a function of $\alpha$ in the log-log plot. The straight line represents a fit to the power law. action and the eigenvalues with the classical results (6.1) and (2.15), respectively.

Next we start the simulation with a particular multi-fuzzy-sphere configuration, and consider its 'life time' defined by the number of sweeps necessary for the state to decay into another state. In practice the 'life time' can be extracted from the history of the action, which has a clear plateau as the ones in Fig. 9 (left). In Fig. 10 we show the 'life time' of the multi-fuzzy-sphere state $\langle 3,5\rangle$ for $N=8$ as a function of $\alpha$ in the log-log plot. Although the 'life time' thus defined is a probabilistic quantity depending on the particular series of random numbers, we see from Fig. 10 that it obeys a power law on the average. The meaning of this observation shall be discussed in the next Section.

\subsection{The 'life time' of the $k$ coincident fuzzy spheres}
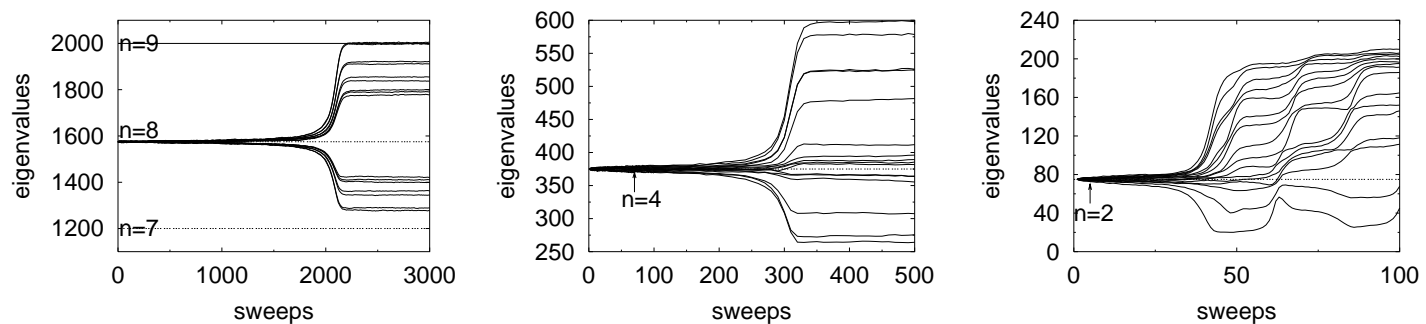

Figure 11: The history of the eigenvalues of the Casimir operator (5.2) is plotted for $N=16$, $\alpha=10.0$. The initial configuration is taken to be the $k$ coincident fuzzy spheres (6.2) with $k=2$ (left), $k=4$ (middle) and $k=8$ (right).

\footnotetext{
${ }^{4}$ At the quantum level the issue becomes more nontrivial; see Section 6.4.
} 
From now on we discuss the properties of the $k$ coincident fuzzy spheres

$$
A_{\mu}=\alpha L_{\mu}^{(n)} \otimes \mathbf{1}_{k}
$$

which correspond to the case $n_{1}=\cdots=n_{k} \equiv n\left(=\frac{N}{k}\right)$ of (2.12). Such a configuration is of particular interest since it gives rise to a NC gauge theory on the fuzzy sphere with the gauge group of rank $k$. From the viewpoint of perturbative calculations, the $k$ coincident fuzzy spheres are easier to handle than more general multi fuzzy spheres, and we have obtained explicit results in the Appendices B and C. Here we perform Monte Carlo simulation with $N=16, \alpha=10.0$ starting from the $k$ coincident fuzzy spheres (6.2) with $k=2,4,8$. Fig. 11 shows the history of the eigenvalues of the Casimir operator.

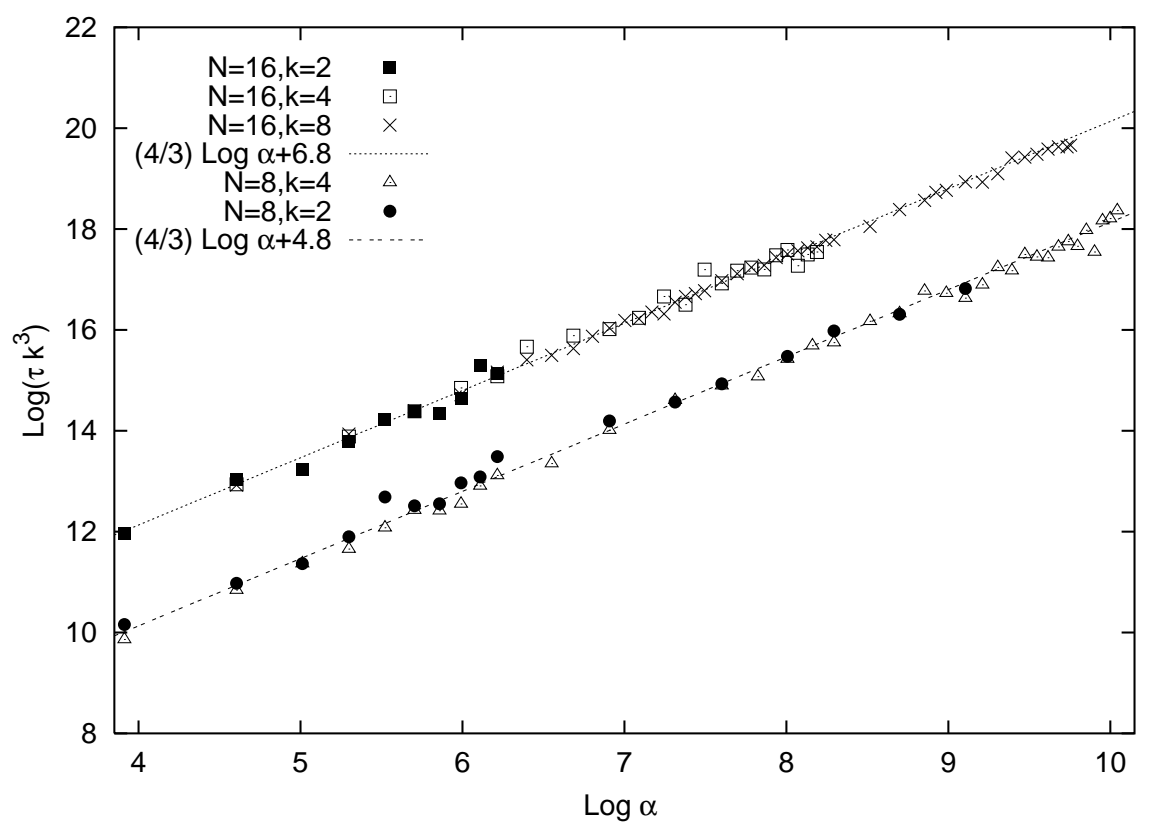

Figure 12: The 'life time' $(\tau)$ of $k$ coincident fuzzy spheres with $k=2,4,8$ follows a universal power law. The plot shows $\log \left(\tau k^{3}\right)$ v.s. $\log \alpha$ for $N=8,16$. The straight lines represent the fits to $\log \left(\tau k^{3}\right)=\frac{4}{3} \log \alpha+c$.

We observe that the $k$ coincident fuzzy spheres also have a finite 'life time', and it becomes shorter for larger $k$. For all $k$ the decay process starts with the spreading of the eigenvalues. We have continued the runs shown in Fig. 11 until the configurations finally thermalize. After visiting various multi fuzzy spheres on the way, all the simulations starting with different $k$ end up with the single fuzzy sphere, the final stage looking very much like Fig. 9. The 'life time' $\tau$ can be extracted from the plateau in the history of the action as in the previous Section. In Fig. 12 we plot $\log \left(\tau k^{3}\right)$ against $\log \alpha$, which reveals a power law

$$
\tau \propto \alpha^{\frac{4}{3}} k^{-3}
$$

for both $N=8$ and $N=16$ over a huge range of $\alpha(\alpha=50 \sim 20000)$. For a different algorithm, the 'life time' will be multiplied by some constant which is naively expected to 
be independent of both $\alpha$ and $k$. If that is the case, the powers obtained here should have some universal meaning.

This result is in striking contrast to the naive expectation that the decay probability $P$ is given by $P \sim \mathrm{e}^{-S}=\exp \left(-\right.$ const. $\left.\frac{N^{4} \alpha^{4}}{k^{2}}\right)$ and the 'life time' is given by its inverse. We interpret it as a consequence of certain instability as discussed in the Appendix D.

The power law observed for a more general multi-fuzzy-sphere state $\langle 3,5\rangle$ in the previous Section may be understood as a consequence of the instability for shifting the center of the spheres relatively. This is consistent with the rather smeared distribution of the eigenvalues observed in Fig. 9 (right) as compared with the distribution for the single fuzzy sphere. Such instability, however, may disappear at larger $N$ as discussed for the coincident case in the Appendix D. Then the decay of multi fuzzy spheres will be suppressed exponentially. Indeed at $N=32$ we observed some cases where a multi-fuzzy-sphere state does not decay into other states during the simulations.

\subsection{The one-loop dominance for the $k$ coincident fuzzy spheres}

Although the $k$ coincident fuzzy spheres have a finite 'life time', we can still measure the observables studied in Section 4.1 and calculate the expectation value before the decay actually occurs. The observables can also be calculated within the perturbation theory around the $k$ coincident fuzzy spheres (6.2) neglecting the zero modes (See Appendix C). The leading large $N$ results at the one-loop level is given by (C.9), (C.11), (C.12) and (C.13).

Fig. 13 shows the results for the observables obtained from simulations starting from the $k=2$ coincident fuzzy spheres (6.2). As in the case of the single fuzzy sphere $(k=1)$, we find that all the observables show a discontinuity at some critical point, but this time the critical point is given by

$$
\tilde{\alpha}_{\mathrm{cr}}^{(1) k=2} \sim 2.8
$$

Above the critical point the results agree very well with the one-loop calculation. This suggests that the one-loop dominance holds for $k$ coincident fuzzy spheres as well as for the single fuzzy sphere.

We can also understand the observed value (6.4) for the critical point. As we discussed in Section 5.1, we can put a lower bound on the critical point by assuming the one-loop dominance for the space-time extent $\left\langle\frac{1}{N} \operatorname{tr}\left(A_{\mu}\right)^{2}\right\rangle$. For general $k$ we find a lower bound $\tilde{\alpha}_{\mathrm{cr}}^{(1) k}>\sqrt{2 k}$, which is satisfied by (6.4). In fact from the analysis which leads to the result (4.9) for $k=1$, we obtain

$$
\tilde{\alpha}_{\mathrm{cr}}^{(1) k} \simeq \sqrt{k}\left(\frac{8}{3}\right)^{3 / 4}
$$

at large $N$. For $k=2$ it gives $\tilde{\alpha}_{\mathrm{cr}}^{(1) k=2} \simeq \sqrt{2}\left(\frac{8}{3}\right)^{3 / 4}=2.95$, which is indeed close to (6.4).

\subsection{Comparison of the one-loop effective action}

The instability of the $k$ coincident fuzzy spheres, which seems to be responsible for the finite 'life time' obeying the power law, may disappear at larger $N$ as discussed in the Appendix 

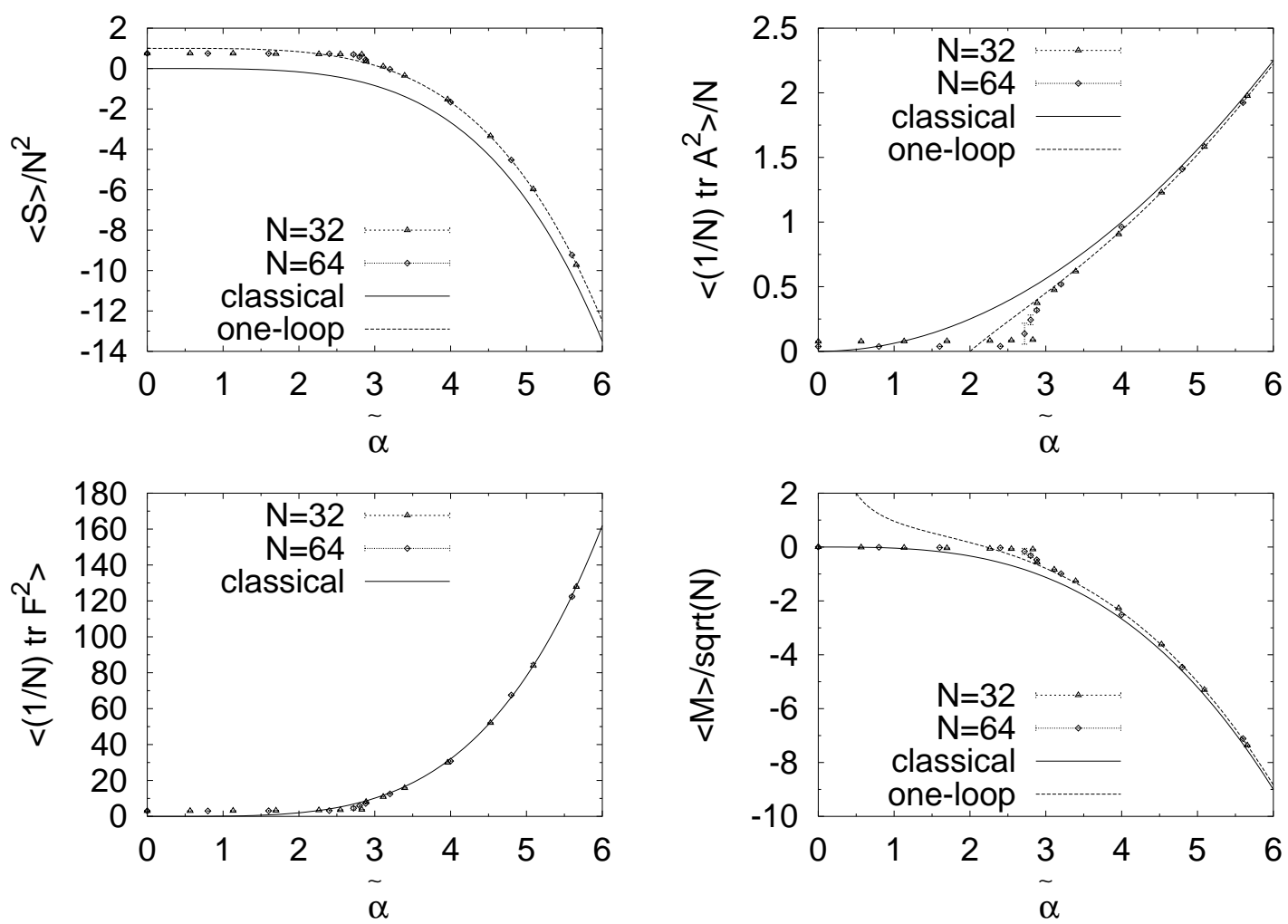

Figure 13: Various observables are plotted against $\tilde{\alpha}$ for $N=32,64$. The initial configuration is taken to be the $k=2$ coincident fuzzy spheres (6.2), and the measurement has been made before they decay into other states.

D. Therefore by Monte Carlo simulation alone, we cannot exclude the possibility that the $k$ coincident fuzzy spheres become the true vacuum of the model at some value of $\tilde{\alpha}$ in the large $N$ limit. Assuming the one-loop dominance, however, we may discuss this issue rigorously by using the one-loop effective action, which is derived in the Appendix B. By comparing (B.17) and (B.23) we find that the $k$ coincident fuzzy spheres have a smaller effective action than the single fuzzy sphere for

$$
\tilde{\alpha}<\left\{\frac{24 \log k}{1-\frac{1}{k^{2}}}\right\}^{1 / 4} \equiv \tilde{\alpha}_{k} .
$$

The critical value $\tilde{\alpha}_{k}$ is 2.17 for $k=2$, and it grows very slowly with $k$.

Here the existence of the 'lower critical point' (6.5) for the $k$ coincident fuzzy spheres has a dramatic consequence. Note that the 'lower critical point' $\tilde{\alpha}_{\mathrm{cr}}^{(1) k} \simeq 2.1 \sqrt{k}$ grows much faster with $k$ than $\tilde{\alpha}_{k}$. As a result it turns out that $\tilde{\alpha}_{k}<\tilde{\alpha}_{\mathrm{cr}}^{(\mathrm{l}) k}$ for any $k \geq 2$. This means that all the $k(\geq 2)$ coincident fuzzy spheres are actually taken over by the Yang-Mills phase at $\tilde{\alpha}$ where they could have a smaller effective action than the single fuzzy sphere. Thus we conclude that the $k$ coincident fuzzy spheres cannot be realized as the true vacuum in this model even in the large $N$ limit. 


\section{Summary and discussions}

In this paper we have studied nonperturbative properties of the matrix model with the Chern-Simons term. In particular we have demonstrated that the single fuzzy sphere emerges dynamically for sufficiently large $\alpha$, and it describes the true vacuum of the model in that regime. The model may therefore serve as a nonperturbative definition of a NC gauge theory on the fuzzy sphere. It would be interesting to study various correlation functions from the field theoretical point of view and to study the UV/IR mixing effects arising from noncommutative geometry as has been done for the NC torus [24-26].

The dynamical generation of the fuzzy sphere itself is an interesting phenomenon, since it provides us with a concrete example in which the space-time that appears dynamically has lower dimensionality than the original dimensionality that the model can actually describe. Although the mechanism may be different, our result gives more plausibility to the scenario for the dynamical generation of the four-dimensional space-time in the IKKT matrix model [3-6].

When $\alpha$ is smaller than a critical value, the single fuzzy sphere is no more stable, and the large $N$ behavior of the system is qualitatively the same as in the pure YangMills model $(\alpha=0)$. Here the space-time looks more like a solid ball with higher density toward the center due to the one-loop attractive potential. It would be interesting to study the supersymmetric case where the one-loop attractive potential is canceled by the fermionic contributions at the leading order of $N$. The density distribution actually has an empty region in the center, which can be explained by the 'uncertainty principle', but this picture may depend on how one defines the density distribution when the space-time is noncommutative.

The fuzzy sphere phase and the Yang-Mills phase are separated by a first order phase transition and we observe a strong hysteresis. One of the interesting features of the phase transition is that the lower critical point behaves as $\alpha_{\mathrm{cr}}^{(1)} \sim \frac{2.1}{\sqrt{N}}$, whereas the upper critical point behaves as $\alpha_{\mathrm{cr}}^{(\mathrm{u})} \sim 0.66$ at large $N$. The position of the lower critical point can be reproduced by considering the effective potential for the scalar mode on the fuzzy sphere [70].

Various multi fuzzy spheres appear as meta-stable states at sufficiently large $\alpha$, but we cannot exclude the possibility that they stabilize eventually in the large $N$ limit. We have argued, however, that the $k$ coincident fuzzy spheres cannot be the true vacuum even in the large $N$ limit. This conclusion was obtained from the comparison of the one-loop effective action taking account of the existence of the 'lower critical point' for each $k$.

The quantum corrections around the fuzzy-sphere solutions are found to be dominated by the one-loop contribution at large $N$ despite the absence of supersymmetry. This may be understood by the naive power counting argument as the one in Ref. [9]. It is noteworthy that the 'one-loop dominance' seems to hold even for meta-stable states such as the $k$ coincident fuzzy spheres. Here the measurements in simulations are made before the metastable states actually decay, while the perturbative calculations are performed omitting the zero modes. 
Thanks to the meta-stability of the $k$ coincident fuzzy spheres, the model may serve also as a toy model for the dynamical generation of gauge group in matrix models. In Ref. [78] it was argued that the $\mathrm{U}(k)$ gauge symmetry appears in the low-energy effective theory if the eigenvalues of $A_{\mu}$ form a cluster of size $k$. It would be interesting to examine whether such a clustering is really taking place in the present model at the fully nonperturbative level. From that point of view, it would be also interesting to search for a model in which the gauge group of higher rank is generated in the true vacuum.

We hope that the present work demonstrates the usefulness of Monte Carlo simulations in revealing various interesting dynamics of matrix models associated in particular with the eigenvalues of the matrices. The actual dynamics may of course depend much on the model, and therefore it is important to extend these studies to other models. Of particular interest is the impact of supersymmetry. The supersymmetric extension of the model we studied in this paper is known [59], but the partition function diverges [76] except for the particular case of $N=2$ [75]. A sensible starting point may therefore be to add the three-dimensional Chern-Simons term (as the one in the present model) to the $D=4$ supersymmetric matrix model studied in Ref. [79]. Another direction is to study matrix models which have higher dimensional fuzzy spheres as classical solutions [80-82]. The stability issue in this case has not been studied yet due to technical complications, but the application of our method is straightforward. Extensions to various homogeneous spaces by replacing the epsilon tensor in the Chern-Simons term by the structure constant of more general Lie algebra may also be of interest. Finally we add that studying matrix models with a cubic term may be of relevance to supermatrix models based on super Lie algebra $[81,83]$ as well as to the quantum Hall systems [84].

\section{Acknowledgments}

We would like to thank Maxime Bagnoud, So Matsuura and Yastoshi Takayama for their participation at the earlier stage of this work. We are also grateful to Satoshi Iso, Hikaru Kawai, Noboru Kawamoto, Yusuke Kimura, Yoshihisa Kitazawa, Yoshinobu Kuramashi, Xavier Martin, Denjoe O'Connor, Tetsuya Onogi, Dan Tomino, Badis Ydri and Kentaroh Yoshida for helpful discussions. The work of T.A., S.B. and J.N. is supported in part by Grant-in-Aid for Scientific Research (Nos. 01282, P02040 and 14740163, respectively) from the Ministry of Education, Culture, Sports, Science and Technology. T.A. and K.N. are greatly benefited from discussions at "YONUPA Summer School 2003" (YITP-W-03-09) and "YITP School on Lattice Field Theory" (YITP-W-02-15) supported by the Yukawa Institute for Theoretical Physics (YITP). Part of the computations were carried out on the supercomputers at YITP.

\section{A. The heat bath algorithm for Monte Carlo simulations}

In this Section we comment on the algorithm used for our Monte Carlo simulation. The crucial point is that the Chern-Simons term is linear with respect to each of $A_{1}, A_{2}$ and 
$A_{3}$. This allows us to extend the heat bath algorithm used for the pure Yang-Mills model $(\alpha=0)$ in Ref. [69] to the present model in a straightforward manner.

Following Ref. [69] we consider the action

$$
S^{\prime}=\frac{N}{2} \sum_{\mu<\nu} \operatorname{tr}\left(Q_{\mu \nu}^{2}-2 Q_{\mu \nu} G_{\mu \nu}\right)+2 N \sum_{\mu<\nu} \operatorname{tr}\left(A_{\mu}^{2} A_{\nu}^{2}\right)+\frac{2 i \alpha N}{3} \epsilon_{\mu \nu \rho} \operatorname{tr} A_{\mu} A_{\nu} A_{\rho}
$$

where $G_{\mu \nu}=\left\{A_{\mu}, A_{\nu}\right\}$. We have introduced the auxiliary fields $Q_{\mu \nu}(1 \leq \mu<\nu \leq 3)$, which are $N \times N$ hermitian matrices. For $\mu>\nu$ we define $Q_{\mu \nu}$ as $Q_{\mu \nu}=Q_{\nu \mu}$. Integrating out the auxiliary fields $Q_{\mu \nu}$, we retrieve the original action (2.2).

The auxiliary fields $\left(Q_{\mu \nu}\right)_{i j}$ can be updated by generating a hermitian matrix with an appropriate Gaussian weight and shifting it by $G_{\mu \nu}$. In order to consider how to update $A_{\rho}$, we extract the part of the action (A.1) depending on $A_{\rho}$ as

$$
S^{\prime}=2 N \operatorname{tr}\left\{S_{\rho}\left(A_{\rho}\right)^{2}\right\}-N \operatorname{tr}\left(T_{\rho} A_{\rho}\right)+\cdots,
$$

where $S_{\rho}$ and $T_{\rho}$ are hermitian matrices defined by

$$
\begin{aligned}
& S_{\rho}=\sum_{\mu \neq \rho}\left(A_{\mu}\right)^{2} \\
& T_{\rho}=\sum_{\mu \neq \rho}\left(A_{\mu} Q_{\rho \mu}+Q_{\rho \mu} A_{\mu}\right)-2 i \alpha \sum_{\mu, \nu} \epsilon_{\rho \mu \nu} A_{\mu} A_{\nu} .
\end{aligned}
$$

Since $\left(A_{\rho}\right)_{i j}$ couples only with $\left(A_{\rho}\right)_{j k}$, where the index $j$ is common, we can update $\frac{N}{2}$ components of $A_{\rho}$, which do not have common indices, simultaneously. The only modification needed to incorporate the Chern-Simons term is the second term in (A.4).

\section{B. The one-loop effective action}

In this Section we formulate the perturbation theory and derive the one-loop effective action for the single fuzzy sphere and the $k$ coincident fuzzy spheres, which is discussed in Section 6.4.

We decompose $A_{\mu}$ into the classical background $X_{\mu}$ and the fluctuation $\tilde{A}_{\mu}$ as

$$
A_{\mu}=X_{\mu}+\tilde{A}_{\mu}
$$

and integrate over $\tilde{A}_{\mu}$ perturbatively. In order to remove the zero modes associated with the $\mathrm{SU}(N)$ invariance, we introduce the gauge fixing term and the corresponding ghost term

$$
\begin{aligned}
S_{\text {g.f. }} & =-\frac{N}{2} \operatorname{tr}\left[X_{\mu}, A_{\mu}\right]^{2}, \\
S_{\text {ghost }} & =-N \operatorname{tr}\left(\left[X_{\mu}, \bar{c}\right]\left[A_{\mu}, c\right]\right),
\end{aligned}
$$

where $c$ and $\bar{c}$ are the ghost and anti-ghost fields, respectively. The total action reads

$$
S_{\text {total }}=S+S_{\text {g.f. }}+S_{\text {ghost }}
$$


which is given explicitly as

$$
\begin{aligned}
S_{\text {total }}= & S[X]+S_{\text {kin }}+S_{\text {int }}, \\
S_{\text {kin }}= & \frac{1}{2} N \operatorname{tr}\left(\tilde{A}_{\mu}\left[X_{\lambda},\left[X_{\lambda}, \tilde{A}_{\mu}\right]\right]\right)+N \operatorname{tr}\left(\bar{c}\left[X_{\lambda},\left[X_{\lambda}, c\right]\right]\right) \\
& -N \operatorname{tr}\left\{\left(\left[X_{\mu}, X_{\nu}\right]-i \alpha \epsilon_{\mu \nu \rho} X_{\rho}\right)\left[\tilde{A}_{\mu}, \tilde{A}_{\nu}\right]\right\}, \\
S_{\text {int }}= & -N \operatorname{tr}\left(\left[\tilde{A}_{\mu}, \tilde{A}_{\nu}\right]\left[X_{\mu}, \tilde{A}_{\nu}\right]\right)-\frac{1}{4} N \operatorname{tr}\left(\left[\tilde{A}_{\mu}, \tilde{A}_{\nu}\right]^{2}\right) \\
& +\frac{2}{3} i \alpha \epsilon_{\mu \nu \rho} N \operatorname{tr}\left(\tilde{A}_{\mu} \tilde{A}_{\nu} \tilde{A}_{\rho}\right)+N \operatorname{tr}\left(\bar{c}\left[X_{\mu},\left[\tilde{A}_{\mu}, c\right]\right]\right) .
\end{aligned}
$$

The linear terms in $X_{\mu}$ cancel since $X_{\mu}$ is assumed to satisfy the classical equation of motion (2.3). Here and henceforth we restrict ourselves to the case where the classical solution $X_{\mu}$ is proportional to $\alpha$. Then, by rescaling the matrices as $A_{\mu} \mapsto \alpha A_{\mu}, c \mapsto \alpha c$, $\bar{c} \mapsto \alpha \bar{c}$, all the terms in the total action $S_{\text {total }}$ will be proportional to $\alpha^{4}$. This means that the expansion parameter of the present perturbation theory is $\frac{1}{\alpha^{4}}$.

The effective action $W$ is defined by

$$
\mathrm{e}^{-W}=\int \mathrm{d} \tilde{A} \mathrm{~d} c \mathrm{~d} \bar{c} \mathrm{e}^{-S_{\text {total }}}
$$

and we calculate it as a perturbative expansion $W=\sum_{j=0}^{\infty} W_{j}$, where $W_{j}=\mathrm{O}\left(\alpha^{4(1-j)}\right)$. The classical part is given by $W_{0}=S[X]$, which is nothing but the action (2.2) evaluated at the classical solution $A_{\mu}=X_{\mu}$.

In this Section we consider the solutions of the form (2.6), for which the third term in (B.6) vanishes. ${ }^{5}$ Then the kinetic term (B.6) can be written as

$$
S_{\text {kin }}=N \alpha^{2} \operatorname{tr}\left[\frac{1}{2} \tilde{A}_{\mu}\left(\mathcal{P}_{\lambda}\right)^{2} \tilde{A}_{\mu}+\bar{c}\left(\mathcal{P}_{\lambda}\right)^{2} c\right]
$$

where we have introduced the operator $\mathcal{P}_{\mu}$

$$
\mathcal{P}_{\mu} M \equiv\left[X_{\mu}, M\right]
$$

which acts on the space of $N \times N$ matrices. The one-loop term can be obtained as

$$
W_{1}=\frac{1}{2} \mathcal{T} r \log \left\{N\left(\mathcal{P}_{\mu}\right)^{2}\right\}
$$

where the symbol $\mathcal{T r}$ denotes the trace of such an operator.

\section{B.1 The single fuzzy sphere}

Let us first consider the single fuzzy sphere $X_{\mu}=\alpha L_{\mu}^{(N)}$. The classical part is given by

$$
W_{0}=-\frac{1}{24} \alpha^{4} N^{2}\left(N^{2}-1\right)
$$

\footnotetext{
${ }^{5}$ We will consider a more general case in Appendix D.
} 
In order to calculate the one-loop term, we note that the eigenvalue problem of the operator $\left(\mathcal{P}_{\lambda}\right)^{2}$ in the present case can be solved by the matrix analog of the spherical harmonics $Y_{l m}(0 \leq l \leq N-1,-l \leq m \leq l)$, which form a complete basis in the space of $N \times N$ matrices and have the property

$$
\left(\mathcal{P}_{\mu}\right)^{2} Y_{l m}=\alpha^{2} l(l+1) Y_{l m} .
$$

Similarly to the usual spherical harmonics, they satisfy

$$
\begin{aligned}
\frac{1}{N} \operatorname{tr}\left(Y_{l m}^{\dagger} Y_{l^{\prime} m^{\prime}}\right) & =\delta_{l l^{\prime}} \delta_{m m^{\prime}}, \\
Y_{l m}^{\dagger} & =(-1)^{m} Y_{l,-m} .
\end{aligned}
$$

See Ref. [59] for other properties. From (B.13) we find that $Y_{00}=\mathbf{1}_{N}$ is a zero mode, which corresponds to the invariance of the action (2.2) under $A_{\mu} \mapsto A_{\mu}+\alpha_{\mu} \mathbf{1}_{N}$. However,

we should omit this mode when we take the trace $\mathcal{T} r$ in (B.11) since all the matrices $\tilde{A}_{\mu}$, $c$ and $\bar{c}$ are supposed to be traceless. Thus we obtain the one-loop contribution as

$$
W_{1}=\frac{1}{2} \sum_{l=1}^{N-1}(2 l+1) \log \left[N \alpha^{2} l(l+1)\right] .
$$

At large $N$ the one-loop effective action is obtained as

$$
W_{1-\text { loop }} \simeq N^{2}\left(-\frac{\tilde{\alpha}^{4}}{24}+\log \tilde{\alpha}+\log N\right)
$$

where $\tilde{\alpha}=\alpha \sqrt{N}$.

\section{B.2 The $k$ coincident fuzzy spheres}

Next we consider the $k$ coincident fuzzy spheres

$$
X_{\mu}=\alpha L_{\mu}^{(n)} \otimes \mathbf{1}_{k}
$$

By setting $n_{1}=\cdots=n_{k} \equiv n\left(=\frac{N}{k}\right)$ in $(6.1)$, the classical part is obtained as

$$
W_{0}=-\frac{\alpha^{4} N^{2}}{24}\left(n^{2}-1\right)
$$

In order to solve the eigenvalue problem of the operator $\left(\mathcal{P}_{\lambda}\right)^{2}$ in the present case, we consider the $n \times n$ version of the matrix spherical harmonics $Y_{l m}^{\prime}$ and introduce a $k \times k$ matrix $\mathbf{e}^{(a, b)}$, whose $(a, b)$ element is 1 and all the other elements are zero. Then as a complete basis of $N \times N$ matrices, we define

$$
Y_{l m}^{(a, b)} \equiv Y_{l m}^{\prime} \otimes \mathbf{e}^{(a, b)},
$$

which has the property

$$
\left(\mathcal{P}_{\lambda}\right)^{2} Y_{l m}^{(a, b)}=\alpha^{2} l(l+1) Y_{l m}^{(a, b)}
$$


Here $Y_{00}^{(a, b)}$ for all the $(a, b)$ blocks are the zero modes. The trace part $\sum_{a=1}^{k} Y_{00}^{(a, a)}=\mathbf{1}_{N}$ should be omitted as before, but not the others. We will study the remaining $\left(k^{2}-1\right)$ zero modes in Appendix D, but here let us consider only the non-zero modes. Then the one-loop contribution $W_{1}$ is obtained as

$$
W_{1}=\frac{1}{2} k^{2} \sum_{l=1}^{n-1}(2 l+1) \log \left[N \alpha^{2} l(l+1)\right] .
$$

At large $N$ the one-loop effective action (neglecting the zero modes) reads

$$
W_{1-\text { loop }} \simeq N^{2}\left(-\frac{\tilde{\alpha}^{4}}{24 k^{2}}+\log \tilde{\alpha}+\log \frac{N}{k}\right) .
$$

\section{The one-loop calculation of various observables}

In this Section we apply the perturbation theory discussed in Appendix B to the oneloop calculation of various observables which are studied by Monte Carlo simulations in this paper. Here we take the background to be $k$ coincident fuzzy spheres (B.18), but the results for the single fuzzy sphere can be readily obtained by setting $k=1$. As in Appendix B.2, we omit the zero modes for $k \geq 2$, which will be discussed in Appendix D.

We note that the number of loops in the relevant diagrams can be less than the order

of $\frac{1}{\alpha^{4}}$ in the perturbative expansion since we are expanding the theory around a nontrivial background. At the one-loop level, the only nontrivial task is to evaluate the tadpole $\left\langle\left(\tilde{A}_{\mu}\right)_{i j}\right\rangle$ explicitly.

\section{C.1 Propagators and the tadpole}

Using the properties (B.14), (B.15) of the matrix spherical harmonics, the propagators for $\tilde{A}_{\mu}$ and the ghosts are given as

$$
\begin{aligned}
\left\langle\left(\tilde{A}_{\mu}\right)_{i j}\left(\tilde{A}_{\nu}\right)_{k l}\right\rangle_{0} & =\delta_{\mu \nu} \frac{1}{n} \sum_{a b} \sum_{l=1}^{n-1} \sum_{m=-l}^{l} \frac{(-1)^{m}}{N \alpha^{2} l(l+1)}\left(Y_{l m}^{(a, b)}\right)_{i j}\left(Y_{l,-m}^{(b, a)}\right)_{k l}, \\
\left\langle(c)_{i j}(\bar{c})_{k l}\right\rangle_{0} & =\frac{1}{n} \sum_{a b} \sum_{l=1}^{n-1} \sum_{m=-l}^{l} \frac{(-1)^{m}}{N \alpha^{2} l(l+1)}\left(Y_{l m}^{(a, b)}\right)_{i j}\left(Y_{l,-m}^{(b, a)}\right)_{k l},
\end{aligned}
$$

where the symbol $\langle\cdot\rangle_{0}$ refers to the expectation value calculated using the kinetic term $S_{\text {kin }}$ in (B.6) only.

Due to the symmetries, the tadpole $\left\langle\tilde{A}_{\mu}\right\rangle$ can be expressed as

$$
\left\langle\tilde{A}_{\mu}\right\rangle=c X_{\mu}
$$

with some coefficient $c$. Using the identity

$$
\begin{aligned}
\operatorname{tr}\left(X_{\mu}\left\langle\tilde{A}_{\mu}\right\rangle\right) & =c \operatorname{tr}\left(X_{\mu} X_{\mu}\right) \\
& =c \frac{\alpha^{2}}{4}\left(n^{2}-1\right) N,
\end{aligned}
$$


the coefficient $c$ can be determined by calculating the left hand side of (C.4).

At the leading order in $\frac{1}{\alpha^{4}}$, we have

$$
\begin{aligned}
\frac{1}{N} \operatorname{tr}\left(X_{\mu}\left\langle\tilde{A}_{\mu}\right\rangle_{1-\text { loop }}\right)= & \left\langle\operatorname{tr}\left(X_{\mu} \tilde{A}_{\mu}\right) \operatorname{tr}\left(\left[\tilde{A}_{\nu}, \tilde{A}_{\rho}\right]\left[X_{\nu}, \tilde{A}_{\rho}\right]\right)\right\rangle_{0} \\
& -\left\langle\operatorname{tr}\left(X_{\mu} \tilde{A}_{\mu}\right) \operatorname{tr}\left(\frac{2}{3} i \alpha \epsilon_{\nu \rho \sigma} \tilde{A}_{\nu} \tilde{A}_{\rho} \tilde{A}_{\sigma}\right)\right\rangle_{0} \\
& -\left\langle\operatorname{tr}\left(X_{\mu} \tilde{A}_{\mu}\right) \operatorname{tr}\left(\bar{c}\left[X_{\nu},\left[\tilde{A}_{\nu}, c\right]\right]\right)\right\rangle_{0} .
\end{aligned}
$$

Using the fact that $X_{\mu}$ is a linear combination of $\left(Y_{l=1, m}^{\prime} \otimes \mathbf{1}_{k}\right)$, we can calculate (C.5) in a straightforward manner. After some algebra we arrive at

$$
\operatorname{tr}\left(X_{\mu}\left\langle\tilde{A}_{\mu}\right\rangle_{1-\text { loop }}\right)=-\frac{k^{2}}{2 N \alpha^{2}}\left(n^{2}-1\right) .
$$

Using (C.4) we obtain

$$
\left\langle\tilde{A}_{\mu}\right\rangle_{1-\text { loop }}=-\frac{2 k^{2}}{N^{2} \alpha^{4}} X_{\mu}
$$

\section{C.2 One-loop results for various observables}

Using the propagator and the tadpole obtained in the previous Section, we can evaluate various observables easily at the one-loop level.

The two-point function $\left\langle\frac{1}{N} \operatorname{tr}\left(A_{\mu}\right)^{2}\right\rangle$ can be evaluated as

$$
\begin{aligned}
\left\langle\frac{1}{N} \operatorname{tr}\left(A_{\mu}\right)^{2}\right\rangle_{1-\text { loop }} & =\frac{1}{N}\left[\operatorname{tr}\left(X_{\mu} X_{\mu}\right)+2 \operatorname{tr}\left(X_{\mu}\left\langle\tilde{A}_{\mu}\right\rangle_{1-\text { loop }}\right)+\left\langle\operatorname{tr}\left(\tilde{A}_{\mu}\right)^{2}\right\rangle_{0}\right] \\
& =\alpha^{2}\left[\frac{1}{4}\left(n^{2}-1\right)-\frac{1}{\alpha^{4}}\left(1-\frac{1}{n^{2}}\right)+\frac{3}{\alpha^{4} n^{2}} \sum_{l=1}^{n-1} \frac{2 l+1}{l(l+1)}\right] .
\end{aligned}
$$

At large $N$ with fixed $\tilde{\alpha}=\sqrt{N} \alpha$, we obtain

$$
\frac{1}{N}\left\langle\frac{1}{N} \operatorname{tr}\left(A_{\mu}\right)^{2}\right\rangle_{1-\text { loop }} \simeq \tilde{\alpha}^{2}\left[\frac{1}{4 k^{2}}-\frac{1}{\tilde{\alpha}^{4}}\right] .
$$

The Chern-Simons term $\langle M\rangle$ can be evaluated as

$$
\begin{aligned}
\langle M\rangle_{1-\text { loop }} & =\frac{2 i}{3 N} \epsilon_{\mu \nu \rho}\left[\operatorname{tr}\left(X_{\mu} X_{\nu} X_{\rho}\right)+3 \operatorname{tr}\left(X_{\mu} X_{\nu}\left\langle\tilde{A}_{\rho}\right\rangle_{1-\text { loop }}\right)\right] \\
& =-\frac{\alpha^{3}}{6}\left(n^{2}-1\right)+\frac{1}{\alpha}\left(1-\frac{1}{n^{2}}\right) .
\end{aligned}
$$

At large $N$ with fixed $\tilde{\alpha}=\sqrt{N} \alpha$, we get

$$
\frac{1}{\sqrt{N}}\langle M\rangle_{1-\text { loop }} \simeq-\frac{\tilde{\alpha}^{3}}{6 k^{2}}+\frac{1}{\tilde{\alpha}} .
$$

The observable $\left\langle\frac{1}{N} \operatorname{tr} F^{2}\right\rangle$ can be calculated in a similar manner, but we can also deduce it from the exact result (4.11) as

$$
\left\langle\frac{1}{N} \operatorname{tr}\left(F_{\mu \nu}\right)^{2}\right\rangle_{1-\text { loop }}=3\left(1-\frac{1}{N^{2}}\right)-3 \alpha\langle M\rangle_{1-\text { loop }} \simeq \frac{\tilde{\alpha}^{4}}{2 k^{2}} .
$$


Combining (C.10) and (C.12), we get

$$
\frac{1}{N^{2}}\langle S\rangle_{1-\mathrm{loop}} \simeq-\frac{\tilde{\alpha}^{4}}{24 k^{2}}+1
$$

Next we calculate the variance $\sigma^{2}$ of the eigenvalue distribution $f(x)$ of the Casimir operator studied in Section 5. The first term in (5.6) can be evaluated as follows.

$$
\begin{aligned}
\frac{1}{N}\left\langle\operatorname{tr}\left(A_{\mu}{ }^{2}\right)^{2}\right\rangle_{1-\text { loop }}= & \frac{1}{N}\left[\operatorname{tr}\left(X_{\mu}{ }^{2} X_{\nu}{ }^{2}\right)+2 \operatorname{tr}\left(X_{\mu}{ }^{2}\left\{X_{\nu},\left\langle\tilde{A}_{\nu}\right\rangle_{1-\text { loop }}\right\}\right)\right. \\
& +\operatorname{tr}\left(\left\{X_{\mu}{ }^{2},\left\langle\tilde{A}_{\nu}{ }^{2}\right\rangle_{0}\right\}\right)+2 \operatorname{tr}\left(X_{\nu} X_{\mu}\left\langle\tilde{A}_{\mu} \tilde{A}_{\nu}\right\rangle_{0}\right) \\
& \left.+\left\langle\operatorname{tr}\left(X_{\mu} \tilde{A}_{\mu} X_{\nu} \tilde{A}_{\nu}\right)\right\rangle_{0}+\left\langle\operatorname{tr}\left(\tilde{A}_{\mu} X_{\mu} \tilde{A}_{\nu} X_{\nu}\right)\right\rangle_{0}\right] \\
= & \frac{\left(n^{2}-1\right)^{2}}{16} \alpha^{4}-\frac{\left(n^{2}-1\right)^{2}}{2 n^{2}} \\
& +\left(1-\frac{1}{n^{2}}\right)\left[\frac{5}{2} \sum_{l=1}^{n-1} \frac{2 l+1}{l(l+1)}-1\right] .
\end{aligned}
$$

The second term in (5.6) is given by the square of (C.8). The classical part of $\sigma^{2}$ cancels exactly as it should. Note that since the leading $\mathrm{O}\left(N^{2}\right)$ terms in the one-loop part also cancel, we have to keep track of the subleading $\mathrm{O}(\log N)$ terms. Using the asymptotic behavior

$$
\sum_{l=1}^{n-1} \frac{2 l+1}{l(l+1)} \simeq 2 \log n
$$

at large $n$, we obtain

$$
\left(\sigma^{2}\right)_{1-\text { loop }} \simeq 2 \log \left(\frac{N}{k}\right)
$$

\section{C.3 An alternative derivation}

Since $\operatorname{tr} F^{2}$ and $M$ are the operators that appear in the action $S$, we can obtain their expectation values easily by using the effective action calculated for the $k$ coincident fuzzy sphere in Appendix B.2. Let us consider the action

$$
S\left(\beta_{1}, \beta_{2}, \alpha\right)=N \operatorname{tr}\left(-\frac{\beta_{1}}{4}\left[A_{\mu}, A_{\nu}\right]^{2}+\frac{2 i \alpha \beta_{2}}{3} \epsilon_{\mu \nu \rho} A_{\mu} A_{\nu} A_{\rho}\right),
$$

where we have introduced two free parameters $\beta_{1}$ and $\beta_{2}$, and define the corresponding effective action by

$$
\mathrm{e}^{-W\left(\beta_{1}, \beta_{2}, \alpha\right)}=\int \mathrm{d} A \mathrm{e}^{-S\left(\beta_{1}, \beta_{2}, \alpha\right)}
$$

Then $\left\langle\operatorname{tr}\left(F_{\mu \nu}\right)^{2}\right\rangle$ and $\langle M\rangle$ can be obtained by

$$
\begin{aligned}
\left\langle\frac{1}{N} \operatorname{tr}\left(F_{\mu \nu}\right)^{2}\right\rangle & =\left.\frac{4}{N^{2}} \frac{\partial W}{\partial \beta_{1}}\right|_{\beta_{1}=\beta_{2}=1}, \\
\langle M\rangle & =\left.\frac{1}{\alpha N^{2}} \frac{\partial W}{\partial \beta_{2}}\right|_{\beta_{1}=\beta_{2}=1} .
\end{aligned}
$$


By rescaling the integration variables as $A_{\mu} \mapsto \beta_{1}^{-\frac{1}{4}} A_{\mu}$, we find

$$
W\left(\beta_{1}, \beta_{2}, \alpha\right)=\frac{3}{4}\left(N^{2}-1\right) \log \beta_{1}+W\left(1,1, \alpha \beta_{1}^{-\frac{3}{4}} \beta_{2}\right) .
$$

Using the one-loop result

$$
W(1,1, \alpha)_{1-\text { loop }}=-\frac{\alpha^{4} N^{2}}{24}\left(n^{2}-1\right)+\frac{1}{2} k^{2} \sum_{l=1}^{n-1}(2 l+1) \log \left[N \alpha^{2} l(l+1)\right],
$$

which follows from (B.19) and (B.22), we can reproduce (C.10) and (C.12).

\section{Instability of the $k$ coincident fuzzy spheres}

In the Appendix B.2 we encountered zero modes in the one-loop perturbative expansion around $k$ coincident fuzzy spheres. This corresponds to the fact that the action (2.2) does not change up to the second order perturbation by the deformation

$$
A_{\mu}=\alpha L_{\mu}^{(n)} \otimes \mathbf{1}_{k}+\mathbf{1}_{n} \otimes \delta H_{\mu},
$$

where $\delta H_{\mu}(\mu=1,2,3)$ are $k \times k$ hermitian matrices. In fact the change of the action is given by

$$
\delta S=N n \operatorname{tr}_{k}\left(-\frac{1}{4}\left[\delta H_{\mu}, \delta H_{\nu}\right]^{2}+\frac{2}{3} i \alpha \epsilon_{\mu \nu \rho} \delta H_{\mu} \delta H_{\nu} \delta H_{\rho}\right),
$$

where the symbol $\operatorname{tr}_{k}$ refers to the trace of a $k \times k$ matrix. Note that $\delta S$ can always be made negative for small $\delta H_{\mu}$ by choosing its sign appropriately when $\left[\delta H_{\mu}, \delta H_{\nu}\right] \neq 0$. Thus the $k$ coincident fuzzy spheres are actually not a local minimum of the classical action, although this does not exclude the possibility that they stabilize due to quantum effects at finite $\tilde{\alpha}$ in the large $N$ limit.

When $\delta H_{\mu}(\mu=1,2,3)$ commute with each other, $\delta S$ in (D.2) vanishes, which means that such a deformation gives rise to a flat direction. In fact this is due to the fact that the configuration (D.1) in this case satisfies the equation of motion (2.3) for finite $\delta H_{\mu}$. Let us consider whether the $k$ coincident fuzzy spheres are stable against such a deformation quantum mechanically. For that purpose we calculate the one-loop effective action around the deformed configuration. Note first that we can diagonalize $\delta H_{\mu}$ by applying an $\mathrm{SU}(N)$ transformation $A_{\mu} \mapsto U A_{\mu} U^{\dagger}$, where $U$ is of the form $U=\mathbf{1}_{n} \otimes V$ with $V \in \mathrm{SU}(k)$. We may therefore consider a classical solution

$$
X_{\mu}=\alpha\left(L_{\mu}^{(n)} \otimes \mathbf{1}_{k}+\mathbf{1}_{n} \otimes C_{\mu}\right),
$$

where

$$
C_{\mu}=\operatorname{diag}\left(c_{\mu}^{(1)}, \cdots, c_{\mu}^{(k)}\right)
$$

without loss of generality. In order to make the configuration $X_{\mu}$ traceless, the $k \times k$ matrices $C_{\mu}$ should also be traceless.

$$
\operatorname{tr}_{k}\left(C_{\mu}\right)=\sum_{a=1}^{k} c_{\mu}^{(a)}=0
$$


The second term in (D.3) corresponds to shifting each of the $k$ coincident fuzzy spheres, where the three-dimensional shift vector for the $a$-th sphere is given by $\alpha c_{\mu}^{(a)}(a=1, \cdots, k)$.

Let us therefore calculate the one-loop effective action around the classical solution (D.3) as we did for the $k$ coincident fuzzy spheres in Appendix B. The classical part of the effective action is the same as the $C_{\mu}=0$ case (B.19). At the one-loop level, the shifted configuration (D.3) has the same number of zero modes as the $k$ coincident fuzzy spheres. Among them, $(k-1)$ corresponds to changing $c_{\mu}^{(a)}(a=1, \cdots, k)$ under the constraint (D.5), so we do not have to integrate over them in the present context. The remaining $k(k-1)$ modes correspond to the 'noncommutative shifts' (D.1) discussed above, but we simply omit them as we did in the $C_{\mu}=0$ case.

The kinetic term (B.6) in the present case is given by

$$
S_{\text {kin }}=N \alpha^{2} \operatorname{tr}\left[\frac{1}{2} \tilde{A}_{\mu}\left\{\left(\mathcal{L}_{\lambda}+\mathcal{C}_{\lambda}\right)^{2} \delta_{\mu \nu}+2 i \epsilon_{\mu \lambda \nu} \mathcal{C}_{\lambda}\right\} \tilde{A}_{\nu}+\bar{c}\left(\mathcal{L}_{\lambda}+\mathcal{C}_{\lambda}\right)^{2} c\right]
$$

where we introduced the operators $\mathcal{L}_{\mu}$ and $\mathcal{C}_{\mu}$ as

$$
\begin{aligned}
\mathcal{L}_{\mu} M & =\left[\left(L_{\mu}^{(n)} \otimes \mathbf{1}_{k}\right), M\right], \\
\mathcal{C}_{\mu} M & =\left[\left(\mathbf{1}_{n} \otimes C_{\mu}\right), M\right],
\end{aligned}
$$

which act on the space of $N \times N$ matrices. The one-loop term can be given as

$$
\begin{aligned}
W_{1}= & \frac{1}{2} \mathcal{T} r \operatorname{tr}^{\prime} \log \left[N \alpha^{2}\left\{\left(\mathcal{L}_{\lambda}+\mathcal{C}_{\lambda}\right)^{2} \delta_{\mu \rho}+2 i \epsilon_{\mu \nu \rho} \mathcal{C}_{\nu}\right\}\right] \\
& -\mathcal{T} r \log \left[N \alpha^{2}\left\{\left(\mathcal{L}_{\lambda}+\mathcal{C}_{\lambda}\right)^{2}\right\}\right],
\end{aligned}
$$

where the trace $\operatorname{tr}^{\prime}$ is taken over Lorentz indices. We calculate $W_{1}$ as an expansion with respect to $C_{\mu}$ using the formulae

$$
\begin{aligned}
\mathcal{L}_{1} Y_{l m}^{(a, b)} & =\frac{1}{2}\left(b_{l, m+1} Y_{l, m+1}^{(a, b)}+b_{l, m} Y_{l, m-1}^{(a, b)}\right), \\
\mathcal{L}_{2} Y_{l m}^{(a, b)} & =\frac{1}{2 i}\left(b_{l, m+1} Y_{l, m+1}^{(a, b)}-b_{l, m} Y_{l, m-1}^{(a, b)}\right), \\
\mathcal{L}_{3} Y_{l m}^{(a, b)} & =m Y_{l, m}^{(a, b)}, \\
\left(\mathcal{L}_{\lambda}\right)^{2} Y_{l m}^{(a, b)} & =l(l+1) Y_{l, m}^{(a, b)}, \\
\mathcal{C}_{\lambda} Y_{l m}^{(a, b)} & =c_{\lambda}^{(a, b)} Y_{l m}^{(a, b)},
\end{aligned}
$$

where $c_{\rho}^{(a, b)}=c_{\rho}^{(a)}-c_{\rho}^{(b)}$ and $b_{l, m}$ is given by

$$
b_{l, m}=\sqrt{l(l+1)-m(m-1)} .
$$

After some algebra, we get

$$
W_{1}=\frac{1}{2} \mathcal{T} r \log \left[N \alpha^{2}\left(\mathcal{L}_{\lambda}\right)^{2}\right]+\kappa \sum_{a b}\left(c_{\rho}^{(a, b)}\right)^{2}+\mathrm{O}\left(C_{\rho}^{3}\right)
$$


The first term in (D.16) is nothing but the result (B.22) for the $k$ coincident fuzzy spheres. The coefficient $\kappa$ is given by

$$
\kappa=\frac{1}{6} \sum_{l=1}^{n-1} \frac{2 l+1}{l(l+1)}-2\left(1-\frac{1}{n^{2}}\right) .
$$

Note that the first term in (D.17) grows as $\frac{1}{3} \log n$ at large $n$. By performing the sum in (D.17) numerically, we find that the coefficient $\kappa$ changes its sign from negative to positive at $n=374$.

For the cases studied in Section 6.2, where $n=2,4,8$, the $k$ coincident fuzzy spheres are therefore unstable for such a shift. Note, however, that the instability for the commutative shift is a quantum effect unlike the instability for the non-commutative shift. Since the 'life time' obeys the same power law up to huge values of $\alpha$, we consider that the instability responsible for the observed power law is dominated by the non-commutative shift.

For a more general multi-fuzzy-sphere state $\langle 3,5\rangle$ studied in Section 6.1, on the other hand, we don't have a counterpart of the noncommutative shift. ${ }^{6}$ The observed power law therefore suggests the existence of quantum instability for the commutative shift. Thus the instability for the non-coincident case is expected to be much weaker than the coincident case. This is consistent with our observation that the power " 3.8 " for the former is much larger than $\frac{4}{3}$ for the latter.

\section{References}

[1] N. Ishibashi, H. Kawai, Y. Kitazawa and A. Tsuchiya, "A large-N reduced model as superstring," Nucl. Phys. B 498, 467 (1997) [hep-th/9612115].

[2] H. Aoki, S. Iso, H. Kawai, Y. Kitazawa, A. Tsuchiya and T. Tada, "IIB matrix model," Prog. Theor. Phys. Suppl. 134, 47 (1999) [hep-th/9908038].

[3] H. Aoki, S. Iso, H. Kawai, Y. Kitazawa and T. Tada, "Space-time structures from IIB matrix model", Prog. Theor. Phys. 99 (1998) 713 [hep-th/9802085].

[4] J. Nishimura and G. Vernizzi, "Spontaneous Breakdown of Lorentz Invariance in IIB Matrix Model", JHEP 0004 (2000) 015 [hep-th/0003223];

J. Nishimura and G. Vernizzi, "Brane World Generated Dynamically from String Type IIB Matrices", Phys. Rev. Lett. 85 (2000) 4664 [hep-th/0007022].

[5] K. N. Anagnostopoulos and J. Nishimura, "New approach to the complex-action problem and its application to a nonperturbative study of superstring theory", Phys. Rev. D 66 (2002) 106008 [hep-th/0108041].

[6] J. Nishimura and F. Sugino, "Dynamical generation of four-dimensional space-time in the IIB matrix model", JHEP 0205 (2002) 001 [hep-th/0111102].

[7] H. Kawai, S. Kawamoto, T. Kuroki, T. Matsuo and S. Shinohara, "Mean field approximation of IIB matrix model and emergence of four dimensional space-time", Nucl. Phys. B 647 (2002) 153 [hep-th/0204240];

\footnotetext{
${ }^{6}$ In Ref. [61] the interaction between two fuzzy spheres with different radii has been calculated.
} 
H. Kawai, S. Kawamoto, T. Kuroki and S. Shinohara, "Improved perturbation theory and four-dimensional space-time in IIB matrix model", Prog. Theor. Phys. 109 (2003) 115 [hep-th/0211272].

[8] J. Nishimura, T. Okubo and F. Sugino, "Convergence of the Gaussian expansion method in dimensionally reduced Yang-Mills integrals", JHEP 0210 (2002) 043 [hep-th/0205253];

J. Nishimura, T. Okubo and F. Sugino, "Testing the Gaussian expansion method in exactly solvable matrix models", JHEP 0310 (2003) 057 [hep-th/0309262].

[9] T. Imai, Y. Kitazawa, Y. Takayama and D. Tomino, "Effective actions of matrix models on homogeneous spaces", hep-th/0307007.

[10] J. Ambjørn, K. N. Anagnostopoulos, W. Bietenholz, T. Hotta and J. Nishimura, "Monte Carlo studies of the IIB matrix model at large N," JHEP 0007 (2000) 011 [hep-th/0005147].

[11] Z. Burda, B. Petersson and J. Tabaczek, "Geometry of reduced supersymmetric 4D Yang-Mills integrals," Nucl. Phys. B 602 (2001) 399 [hep-lat/0012001].

[12] J. Ambjørn, K. N. Anagnostopoulos, W. Bietenholz, F. Hofheinz and J. Nishimura, "On the spontaneous breakdown of Lorentz symmetry in matrix models of superstrings," Phys. Rev. D 65 (2002) 086001 [hep-th/0104260].

[13] J. Nishimura, "Exactly solvable matrix models for the dynamical generation of space-time in superstring theory", Phys. Rev. D 65 (2002) 105012 [hep-th/0108070].

[14] G. Vernizzi and J. F. Wheater, "Rotational symmetry breaking in multi-matrix models", Phys. Rev. D 66 (2002) 085024 [hep-th/0206226].

[15] H. Aoki, N. Ishibashi, S. Iso, H. Kawai, Y. Kitazawa and T. Tada, "Noncommutative Yang-Mills in IIB matrix model," Nucl. Phys. B 565, 176 (2000) [hep-th/9908141].

[16] A. Connes, M. R. Douglas and A. Schwarz, "Noncommutative geometry and matrix theory: Compactification on tori," JHEP 9802, 003 (1998) [hep-th/9711162].

[17] A. Connes, Noncommutative geometry, Academic Press, 1990.

[18] R. J. Szabo, "Quantum field theory on noncommutative spaces," Phys. Rept. 378 (2003) 207 [hep-th/0109162].

[19] N. Seiberg and E. Witten, "String theory and noncommutative geometry," JHEP 9909, 032 (1999) [hep-th/9908142].

[20] T. Filk, "Divergencies In A Field Theory On Quantum Space," Phys. Lett. B 376, 53 (1996); S. Minwalla, M. Van Raamsdonk and N. Seiberg, "Noncommutative perturbative dynamics," JHEP 0002 (2000) 020 [hep-th/9912072].

[21] A. Gonzalez-Arroyo and M. Okawa, "The Twisted Eguchi-Kawai Model: A Reduced Model For Large N Lattice Gauge Theory," Phys. Rev. D 27 (1983) 2397.

[22] T. Eguchi and H. Kawai, "Reduction Of Dynamical Degrees Of Freedom In The Large N Gauge Theory," Phys. Rev. Lett. 48 (1982) 1063.

[23] J. Ambjørn, Y. M. Makeenko, J. Nishimura and R. J. Szabo, "Finite N matrix models of noncommutative gauge theory," JHEP 9911 (1999) 029 [hep-th/9911041];

J. Ambjørn, Y. M. Makeenko, J. Nishimura and R. J. Szabo, "Nonperturbative dynamics of noncommutative gauge theory," Phys. Lett. B 480 (2000) 399 [hep-th/0002158];

J. Ambjørn, Y. M. Makeenko, J. Nishimura and R. J. Szabo, "Lattice gauge fields and discrete noncommutative Yang-Mills theory," JHEP 0005 (2000) 023 [hep-th/0004147]. 
[24] W. Bietenholz, F. Hofheinz and J. Nishimura, "The renormalizability of 2D Yang-Mills theory on a non-commutative geometry," JHEP 0209 (2002) 009 [hep-th/0203151].

[25] W. Bietenholz, F. Hofheinz and J. Nishimura, "Simulating non-commutative field theory," Nucl. Phys. Proc. Suppl. 119 (2003) 941 [hep-lat/0209021];

W. Bietenholz, F. Hofheinz and J. Nishimura, "Non-commutative field theories beyond perturbation theory," Fortsch. Phys. 51 (2003) 745 [hep-th/0212258];

W. Bietenholz, F. Hofheinz and J. Nishimura, "The non-commutative $\lambda \phi^{4}$ model," Acta Phys. Polon. B 34 (2003) 4711 [hep-th/0309216];

W. Bietenholz, F. Hofheinz and J. Nishimura, "Numerical results on the non-commutative $\lambda \phi^{4}$ model," hep-th/0309182, to appear in Nucl. Phys. Proc. Suppl.

[26] J. Ambjørn and S. Catterall, "Stripes from (noncommutative) stars," Phys. Lett. B 549 (2002) 253 [hep-lat/0209106].

[27] J. Nishimura, "Lattice superstring and noncommutative geometry," hep-lat/0310019, to appear in Nucl. Phys. Proc. Suppl.

[28] J. Madore, "The fuzzy sphere," Class. Quant. Grav. 9, 69 (1992).

[29] H. Grosse, C. Klimcik and P. Presnajder, "Towards finite quantum field theory in noncommutative geometry," Int. J. Theor. Phys. 35, 231 (1996) [hep-th/9505175];

B. P. Dolan, D. O'Connor and P. Presnajder, "Matrix phi**4 models on the fuzzy sphere and their continuum limits," JHEP 0203, 013 (2002) [hep-th/0109084];

B. P. Dolan, "The spectrum of the Dirac operator on coset spaces with homogeneous gauge fields," JHEP 0305, 018 (2003) [hep-th/0304037];

B. P. Dolan, D. O'Connor and P. Presnajder, "Fuzzy Complex Quadrics and Spheres," hep-th/0312190.

[30] H. Grosse and J. Madore, "A Noncommutative version of the Schwinger model," Phys. Lett. B 283, 218 (1992);

H. Grosse and P. Presnajder, "The Dirac operator on the fuzzy sphere," Lett. Math. Phys. 33, 171 (1995);

H. Grosse, C. Klimcik and P. Presnajder, "Field theory on a supersymmetric lattice," Commun. Math. Phys. 185, 155 (1997) [hep-th/9507074]; "N = 2 superalgebra and non-commutative geometry," hep-th/9603071.

[31] U. Carow-Watamura and S. Watamura, "Chirality and Dirac operator on noncommutative sphere," Commun. Math. Phys. 183, 365 (1997) [hep-th/9605003];

U. Carow-Watamura and S. Watamura, "Noncommutative geometry and gauge theory on fuzzy sphere," Commun. Math. Phys. 212, 395 (2000) [hep-th/9801195].

[32] A. P. Balachandran, T. R. Govindarajan and B. Ydri, "The fermion doubling problem and noncommutative geometry," Mod. Phys. Lett. A 15, 1279 (2000) [hep-th/9911087].

[33] H. Grosse and P. Presnajder, "A treatment of the Schwinger model within noncommutative geometry," hep-th/9805085;

H. Grosse and P. Presnajder, "A Noncommutative Regularization Of The Schwinger Model," Lett. Math. Phys. 46, 61 (1998).

[34] A. P. Balachandran and S. Vaidya, "Instantons and chiral anomaly in fuzzy physics," Int. J. Mod. Phys. A 16, 17 (2001) [hep-th/9910129]. 
[35] P. Presnajder, "The origin of chiral anomaly and the noncommutative geometry," J. Math. Phys. 41, 2789 (2000) [hep-th/9912050].

[36] H. Aoki, S. Iso and K. Nagao, "Chiral anomaly on fuzzy 2-sphere," Phys. Rev. D 67, 065018 (2003) [hep-th/0209137].

[37] H. Neuberger, "Exactly massless quarks on the lattice," Phys. Lett. B 417 (1998) 141 [hep-lat/9707022].

[38] P. H. Ginsparg and K. G. Wilson, "A Remnant Of Chiral Symmetry On The Lattice," Phys. Rev. D 25, 2649 (1982).

[39] M. Lüscher, "Exact chiral symmetry on the lattice and the Ginsparg-Wilson relation", Phys. Lett. B428 (1998) 342 [hep-lat/9802011].

[40] A. P. Balachandran, T. R. Govindarajan and B. Ydri, "The fermion doubling problem and noncommutative geometry. II," hep-th/0006216.

[41] H. Aoki, S. Iso and K. Nagao, "Ginsparg-Wilson relation, topological invariants and finite noncommutative geometry," Phys. Rev. D 67, 085005 (2003) [hep-th/0209223].

[42] K. Nagao, "Matrix model and Ginsparg-Wilson relation," hep-th/0309153, to appear in Nucl. Phys. Proc. Suppl.

[43] J. Nishimura and M. A. Vazquez-Mozo, "Noncommutative chiral gauge theories on the lattice with manifest star-gauge invariance," JHEP 0108, 033 (2001) [hep-th/0107110].

[44] S. Iso and K. Nagao, "Chiral anomaly and Ginsparg-Wilson relation on the noncommutative torus," Prog. Theor. Phys. 109, 1017 (2003) [hep-th/0212284].

[45] T. Fujiwara, K. Nagao and H. Suzuki, "Axial anomaly with the overlap-Dirac operator in arbitrary dimensions," JHEP 0209, 025 (2002) [hep-lat/0208057].

[46] J. Nishimura and M. A. Vazquez-Mozo, "Lattice perturbation theory in noncommutative geometry and parity anomaly in 3D noncommutative QED," JHEP 0301, 075 (2003) [hep-lat/0210017].

[47] W. Bietenholz and J. Nishimura, "Ginsparg-Wilson fermions in odd dimensions, JHEP 0107 (2001) 015 [hep-lat/0012020].

[48] H. Aoki, S. Iso and K. Nagao, "Ginsparg-Wilson Relation and 't Hooft-Polyakov Monopole on Fuzzy 2-Sphere", hep-th/0312199.

[49] B. Ydri, "Noncommutative chiral anomaly and the Dirac-Ginsparg-Wilson operator," JHEP 0308 (2003) 046 [hep-th/0211209].

[50] H. Grosse, C. Klimcik and P. Presnajder, "Topologically nontrivial field configurations in noncommutative geometry," Commun. Math. Phys. 178, 507 (1996) [hep-th/9510083];

S. Baez, A. P. Balachandran, B. Ydri and S. Vaidya, "Monopoles and solitons in fuzzy physics," Commun. Math. Phys. 208, 787 (2000) [hep-th/9811169];

G. Landi, "Projective modules of finite type and monopoles over $S^{2}$," J. Geom. Phys. 37, 47 (2001) [math-ph/9905014].

[51] P. Valtancoli, "Projectors for the fuzzy sphere," Mod. Phys. Lett. A 16, 639 (2001) [hep-th/0101189].

[52] H. Steinacker, "Quantized gauge theory on the fuzzy sphere as random matrix model," hep-th/0307075. 
[53] A. P. Balachandran and G. Immirzi, "The fuzzy Ginsparg-Wilson algebra: A solution of the fermion doubling problem," Phys. Rev. D 68, 065023 (2003) [hep-th/0301242].

[54] K. Hayasaka, R. Nakayama and Y. Takaya, "A new noncommutative product on the fuzzy two-sphere corresponding to the unitary representation of $\mathrm{SU}(2)$ and the Seiberg-Witten map," Phys. Lett. B 553, 109 (2003) [hep-th/0209240];

J. M. Grimstrup, T. Jonsson and L. Thorlacius, "The Seiberg-Witten map on the fuzzy sphere," hep-th/0310179.

[55] R. C. Myers, "Dielectric-branes," JHEP 9912, 022 (1999) [hep-th/9910053].

[56] A. Y. Alekseev, A. Recknagel and V. Schomerus, "Brane dynamics in background fluxes and non-commutative geometry," JHEP 0005 (2000) 010 [hep-th/0003187].

[57] A. Y. Alekseev, A. Recknagel and V. Schomerus, JHEP 9909 (1999) 023 [hep-th/9908040].

[58] K. Hashimoto and K. Krasnov, "D-brane solutions in non-commutative gauge theory on fuzzy sphere," Phys. Rev. D 64, 046007 (2001) [hep-th/0101145].

[59] S. Iso, Y. Kimura, K. Tanaka and K. Wakatsuki, "Noncommutative gauge theory on fuzzy sphere from matrix model," Nucl. Phys. B 604, 121 (2001) [hep-th/0101102].

[60] Y. Kimura, "Noncommutative gauge theories on fuzzy sphere and fuzzy torus from matrix model," Prog. Theor. Phys. 106, 445 (2001) [hep-th/0103192].

[61] S. Bal and H. Takata, "Interaction between two fuzzy spheres," Int. J. Mod. Phys. A 17, 2445 (2002) [hep-th/0108002].

[62] D. P. Jatkar, G. Mandal, S. R. Wadia and K. P. Yogendran, "Matrix dynamics of fuzzy spheres," JHEP 0201 (2002) 039 [hep-th/0110172].

[63] Y. Kitazawa, "Matrix models in homogeneous spaces," Nucl. Phys. B 642, 210 (2002) [hep-th/0207115].

[64] T. A. Imai, Y. Kitazawa, Y. Takayama and D. Tomino, "Quantum corrections on fuzzy sphere," Nucl. Phys. B 665, 520 (2003) [hep-th/0303120].

[65] Y. J. Zhong and W. H. Huang, "Quantum energy of fuzzy sphere: Gaussian variational method," hep-th/0303196.

[66] D. Berenstein, J. M. Maldacena and H. Nastase, "Strings in flat space and pp waves from N = 4 super Yang Mills," JHEP 0204 (2002) 013 [hep-th/0202021].

[67] K. Dasgupta, M. M. Sheikh-Jabbari and M. Van Raamsdonk, JHEP 0205 (2002) 056 [hep-th/0205185];

K. Sugiyama and K. Yoshida, "Giant graviton and quantum stability in matrix model on PP-wave background," Phys. Rev. D 66 (2002) 085022 [hep-th/0207190];

H. Shin and K. Yoshida, "One-loop flatness of membrane fuzzy sphere interaction in plane-wave matrix model," Nucl. Phys. B 679 (2004) 99 [hep-th/0309258];

W. H. Huang, "Thermal instability of giant graviton in matrix model on pp-wave background," hep-th/0310212;

H. Shin and K. Yoshida, "Thermodynamics of fuzzy spheres in pp-wave matrix model," hep-th/0401014.

[68] P. Valtancoli, "Stability of the fuzzy sphere solution from matrix model," Int. J. Mod. Phys. A 18, 967 (2003) [hep-th/0206075]. 
[69] T. Hotta, J. Nishimura and A. Tsuchiya, "Dynamical aspects of large N reduced models," Nucl. Phys. B 545, 543 (1999) [hep-th/9811220].

[70] D. O'Connor, private communication.

[71] W. Krauth and M. Staudacher, "Finite Yang-Mills integrals," Phys. Lett. B 435 (1998) 350 [hep-th/9804199].

[72] P. Austing and J. F. Wheater, "The convergence of Yang-Mills integrals," JHEP 0102 (2001) 028 [hep-th/0101071].

[73] W. Krauth, H. Nicolai and M. Staudacher, "Monte Carlo approach to M-theory," Phys. Lett. B 431 (1998) 31 [hep-th/9803117].

[74] P. Austing and J. F. Wheater, "Convergent Yang-Mills matrix theories," JHEP 0104 (2001) 019 [hep-th/0103159].

[75] D. Tomino, "N=2 3d-Matrix Integral with Myers Term," hep-th/0309264.

[76] P. Austing and J. F. Wheater, "Adding a Myers term to the IIB matrix model," JHEP 0311, 009 (2003) [hep-th/0310170].

[77] W. Krauth and M. Staudacher, "Eigenvalue distributions in Yang-Mills integrals," Phys. Lett. B 453 (1999) 253 [hep-th/9902113].

[78] S. Iso and H. Kawai, "Space-time and matter in IIB matrix model: Gauge symmetry and diffeomorphism," Int. J. Mod. Phys. A 15, 651 (2000) [hep-th/9903217].

[79] J. Ambjorn, K. N. Anagnostopoulos, W. Bietenholz, T. Hotta and J. Nishimura, "Large N dynamics of dimensionally reduced 4D SU(N) super Yang-Mills theory," JHEP 0007, 013 (2000) [hep-th/0003208].

[80] Y. Kimura, "Noncommutative gauge theory on fuzzy four-sphere and matrix model," Nucl. Phys. B 637, 177 (2002) [hep-th/0204256];

Y. Kimura, "On higher dimensional fuzzy spherical branes," Nucl. Phys. B 664, 512 (2003) [hep-th/0301055].

[81] T. Azuma and M. Bagnoud, "Curved-space classical solutions of a massive supermatrix model," Nucl. Phys. B 651, 71 (2003) [hep-th/0209057].

[82] T. Imai and Y. Takayama, "Stability of fuzzy $\mathrm{S}^{* *} 2 \times \mathrm{S}^{* *} 2$ geometry in IIb matrix model," hep-th/0312241.

[83] L. Smolin, "M theory as a matrix extension of Chern-Simons theory," Nucl. Phys. B 591 (2000) 227 [hep-th/0002009];

L. Smolin, "The cubic matrix model and a duality between strings and loops," hep-th/0006137;

T. Azuma, S. Iso, H. Kawai and Y. Ohwashi, "Supermatrix models," Nucl. Phys. B 610 (2001) 251 [hep-th/0102168];

T. Azuma, "Investigation of matrix theory via super Lie algebra," hep-th/0103003;

Y. Ohwashi, "E(6) matrix model," Prog. Theor. Phys. 108 (2002) 755 [hep-th/0110106];

T. Azuma and H. Kawai, "Matrix model with manifest general coordinate invariance," Phys. Lett. B 538 (2002) 393 [hep-th/0204078];

M. Bagnoud, L. Carlevaro and A. Bilal, "Supermatrix models for M-theory based on osp(1|32,R)," Nucl. Phys. B 641 (2002) 61 [hep-th/0201183]; 
S. Iso and H. Umetsu, "Gauge theory on noncommutative supersphere from supermatrix model," hep-th/0311005;

S. Iso and H. Umetsu, "Note on Gauge Theory on Fuzzy Supersphere," hep-th/0312307.

[84] L. Susskind, "The quantum Hall fluid and non-commutative Chern Simons theory," hep-th/0101029;

A. P. Polychronakos, "Quantum Hall states as matrix Chern-Simons theory," JHEP 0104 (2001) 011 [hep-th/0103013];

E. Fradkin, V. Jejjala and R. G. Leigh, "Non-commutative Chern-Simons for the Quantum Hall System and Duality," Nucl. Phys. B 642 (2002) 483 [cond-mat/0205653]. 